\title{
A Distributed Formation Control Scheme with Obstacle Avoidance for Multiagent Systems
}

\author{
Xiaowu Yang $\mathbb{D}^{1}$ and Xiaoping Fan $\mathbb{D}^{1,2}$ \\ ${ }^{1}$ School of Automation, Central South University, Changsha 410075, China \\ ${ }^{2}$ School of Information Technology and Management, Hunan University of Finance and Economics, Changsha 410205, China \\ Correspondence should be addressed to Xiaoping Fan; xpfan@csu.edu.cn
}

Received 6 March 2019; Revised 24 May 2019; Accepted 9 June 2019; Published 23 June 2019

Academic Editor: Javier Moreno-Valenzuela

Copyright ( 2019 Xiaowu Yang and Xiaoping Fan. This is an open access article distributed under the Creative Commons Attribution License, which permits unrestricted use, distribution, and reproduction in any medium, provided the original work is properly cited.

\begin{abstract}
This study considers the problem of formation control for second-order multiagent systems. We propose a distributed nonlinear formation controller where the control input of each follower can be expressed as a product of a nonlinear term that relies on the distance errors under the leader-follower structure. In the leader-follower structure, a small number of agents are assumed to be the leaders, and they are responsible for steering a group of agents to the specific destination, while the rest of the agents are called followers. The stability of the proposed control laws is demonstrated by utilizing the Lyapunov function candidate. To solve the obstacle avoidance problem, the artificial potential approach is employed, and the agents can avoid each possible obstacle successfully without getting stuck in any local minimum point. The control problem of multiagent systems in the presence of unknown constant disturbances is also considered. To attenuate such disturbances, the integral term is introduced, and the static error is eliminated through the proposed PI controller, which makes the system stable; the adaptive controller is designed to reduce the effect of time-varying disturbances. Finally, numerical simulation results are presented to support the obtained theoretical results.
\end{abstract}

\section{Introduction}

Distributed or decentralized coordination control of multiagent systems has received increasing research attention in the control community; it has been widely used in many practical applications, such as unmanned surface vessels, unmanned aerial vehicles, satellite clusters, and military surveillance [17]. The multiagent systems are generally composed of a team of agents, in which all the agents are required to interact with others and then perform various kinds of tasks.

Three main structures are commonly utilized in the existing literature for the formation control of multiagent systems, i.e., leader-follower, behavioral, and virtual structures. The leader-follower structure is widely used because of its simplicity and implementation, in which a small percentage of agents (or multiple leaders) are designated as the leaders, and the rest are called followers. The reference signals for a group of agents is assumed to be available to the designed leaders. In the behavioral structure, the specified behaviors, for example, collision and obstacle avoidance, are first specified for each agent. The agents possess a rigid geometric configuration using a virtual leader to present the formations in the virtual structure, where the virtual leader will never fail. The more advantages and disadvantages, as well as the distinctions among these three structures can be found in [8-10]. Two methods are considered for the formation control of multiagent systems, namely, the centralized [11] and decentralized controllers $[12,13]$, according to the interaction topology of sensing graph. In the centralized controller, each agent is assumed to have access to all the states collected from the central controller. This controller may fail to achieve the control objective in the presence of faults. In the decentralized controller, each agent can achieve its own tasks defined according to the desired formation only based on the local measurements, which might provide reliable control when communication between agents is limited or even unavailable [14]. As discussed above, the decentralized controller devised for each agent under the leader-follower structure 
is considered in this study. The geometrical structure of the desired formation can be defined by inter-distances, relativepositions, and inter-bearings. The distance-constrained formation control methods can be used to fulfill translational and rotational formation maneuvers [15], which can also be used to solve the obstacle avoidance problem; further, relative position constrained formation control methods are used to achieve the translational formation maneuvers [16], and bearing constrained formation control methods are employed to achieve the translational and scaling formation maneuvers [17], according to the properties of invariant of the constraints. Generally, the distance- and bearing-only-based formation control are nonlinear control problems, whereas the relative position-based formation control and bearingbased formation control are linear control problems [18-21]. The consensus patterns of multiagent systems have appeared in the literature [22, 23]. In [11], the authors proposed a class of nonlinear consensus control laws, which were applied in smart buildings, autonomous space satellites, and frequency of power systems, and PI controllers were designed for eliminating the state errors. In [10], distributed distancebased methods are employed to deal with the formation control for first order dynamics multiagent systems. The consensus problem with unknown disturbance is addressed in [24]. The obstacle avoidance problem is not considered in $[10,11]$. Compared with the consensus, the formation control is more challenging as other physical constraints need to be satisfied.

As mentioned, one of the important problems in the formation control of multiagent systems is the requirement to consider acquiring the desired control performance, which is referred to as obstacle avoidance problem. Cooperative obstacle avoidance of multiagent systems needs to be solved in many practical formation controls owing to its difficulty and complexity. To solve this problem, the artificial potential field (APF) (or) repulsive force approaches are applied. Geometrically, the obstacles can be viewed as static agents, which steer all the dynamic agents away from the static agents (obstacles). The obstacles can be modeled as a single particle/point [25], elliptical shapes, and rectangular shapes, in general $[8,13]$. In $[13]$, the distributed control strategy based on an artificial potential function is proposed to address the formation control and obstacle avoidance with the rectangular agents, which solved the obstacle avoidance problem. In [8], the authors investigated the formation control of a group of robots under virtual and behavioral structures, in which a rotational potential field is employed to drive the autonomous mobile agent away from the obstacle without getting stuck in the local minimum points. APF approaches are also utilized in [25] to deal with the obstacle avoidance problem. This study also considers the relative positionbased formation control of multiagent systems in the presence of unknown constant disturbances. As is well known, multiagent systems are generally vulnerable to disturbances, which leads to failure in achieving the control objective. To eliminate the static errors caused by the bounded unknown constant disturbances, PI controllers are usually introduced for obtaining the desired performance when the systems suffer from constant disturbances, because the PI controllers have high-frequency gain and thus filter any noise produced by the input signals. In [11], distributed PI controllers are employed to address the consensus for first- and secondorder dynamics of systems, which can attenuate the static errors caused by the unknown disturbances. In [17], using PI controllers, the authors proposed bearing-constrained formation control in the presence of input disturbances. Other practical problems such as acceleration saturation, and collision avoidance are also considered in that study. A similar idea was adopted in this study.

As discussed above, formation control with obstacle avoidance problems for second-order dynamics of multiagent systems under the leader-follower control structure is addressed, and the formation control under the constant disturbances are also considered in this study. The main contributions are stated as follows.

(a) The distributed control scheme for achieving and maintaining the desired formation is presented, which only depends on the local measurements, where the nonlinear interaction weights are employed to characterize the convergent behavior for agents appropriately.

(b) The obstacle avoidance problem can be solved by employing the artificial potential function. The agents cannot get stuck into the local minimum points with the assistance of the navigation function, i.e., the attraction function created by the following agents and the leader agents, where the leader agents are assumed to have access to the reference signal for a team of agents.

(c) Multiagent systems are susceptible to various disturbances, such as constant- and time-varying disturbances, while the bounded properties of these disturbances are required in general. We focus on the systems with the bounded disturbances in this study, which will cause a drift effect on the formation characterized by the relative measurements. To address this additional issue, we apply the PI controller to attenuate the bounded unknown constant disturbances, and the static errors can then be eliminated. The control systems with time varying disturbances are also discussed by using the adaptive technique.

The remainder of this paper is organized as follows. In Section 2, the basic concepts of graph theory and artificial potential function are given. The second-order dynamics of multiagent (or system model) are defined in Section 3. The distributed control algorithms for formation control of a multiagent system and artificial potential function approaches for obstacle avoidance of the agents based on leader-follower structure are proposed, respectively. The practical issue of constant disturbances for multiagent systems is studied by using the integral control action in Section 4. In Section 5, the simulation results are provided to illustrate the effectiveness of the proposed control strategy, and conclusions are provided in Section 6.

\section{Preliminaries}

Let $G=(V, E)$ denote a directed graph consisting of $n$ vertices (nodes), where $V=\left\{v_{1}, \ldots, v_{n}\right\}$ and $E \subseteq\left\{\left(v_{i}, v_{j}\right) \in V \times V\right\}$ containing the ordered pairs of vertices are called the node set and edge set, respectively; $M=\left[m_{i j}\right]_{n \times n}$ is the weighted 
adjacency matrix. Each agent can be abstracted as a node in the graph. The edge $e_{i j}=\left(v_{i} ; v_{j}\right)$ is created if the position information of agent $j$ is available to agent $i$, which implies agent $j$ is a neighbor of agent $i$; thus, the interconnection topology of a multiagent system can be modeled as a graph. The information interaction weights among the agents are characterized by the adjacency matrix $M=\left[m_{i j}\right]_{n \times n}$. Note that $m_{i j}>0$ if $e_{i j} \in E$, otherwise $m_{i j}=0$. Furthermore, the possibility of a self-loop is not considered, by convention; i.e., $m_{i i}=0$. The property of time-invariance of $G$ is assumed.

In view of $M$, the diagonal matrix $D=\left\{\sigma_{1}, \cdots, \sigma_{N}\right\}$ can be easily obtained, i.e., each entry $\sigma_{i}$ is calculated as the sum of $i$-th row of $M$. Laplacian matrix of the directed graph is then given as

$$
L=D-M
$$

From (1), one obtains $l_{i i}>0$ and $l_{i j}<0, l_{i i}, l_{i j} \in L$.

The properties of the interaction topology of a graph are characterized by the Laplacian matrix. Note that the Laplacian matrix has the following useful properties:

(i) $L \mathbf{1}=\mathbf{0}$, where $\mathbf{1}_{n}=[1, \ldots, 1]^{T}$ and $\mathbf{0}_{n}=[0, \ldots, 0]^{T}$.

(ii) only one eigenvalue is equal to 0 , which is also referred to as the trivial eigenvalue. The real part of the rest of eigenvalues are positive.

(iii) $L$ is a positive semidefinite.

With the above properties at hand, the following result is easily obtained; namely, the graph is connected if the smallest eigenvalue of $L$ is equal to zero, which is a straightforward consequence according to the property (ii).

To achieve and maintain the desired formation, we further assume that the followers have access to the local information from the leaders under the leader-follower structure. We denote the weight matrix of interaction topology as $\Delta_{L}=$ $\operatorname{diag}\left\{\delta_{1}, \ldots, \delta_{m}\right\}$, where $m$ denotes the cardinality of a set of the leaders with a slight abuse of notion, and the sum of the diagonal entries of $\Delta_{L}$ is greater than zero, i.e., $\delta_{1}+\delta_{2}+\ldots+$ $\delta_{m}>0$. It is observed that the size of $L$ equals to size of $\Delta_{L}$, i.e., $\operatorname{size}(L)=\operatorname{size}\left(\Delta_{L}\right)$. We choose $m \leq 0.2 n$, for the sake of generality, in this study.

According to the properties of Laplacian $L$ and weight matrix $\Delta_{L}$, the following result is easily obtained:

$$
L+\Delta_{L}=\left[\begin{array}{ccc}
l_{11}+\sigma_{1} & \cdots & l_{1 n} \\
\vdots & \ddots & \vdots \\
l_{n 1} & \cdots & l_{n n}+\sigma_{n}
\end{array}\right]
$$

Note that the new matrix $\left(L+\Delta_{L}\right)$ is a positive definite, which is a stable matrix, because all the eigenvalues of $\left(L+\Delta_{L}\right)$ are always positive.

Agent $j$ is said to be a neighbor of agent $i$ if agent $j$ moves to the feasible sensing area of agent $i$ under the assumption that each agent has the limited sensing range $R_{s}$. The neighborhood of $x$ is defined as $\Omega_{z}:=\left\{p \in R^{n d} \mid\right.$ $\|p-x\|<\epsilon\}$ with $\Omega_{x}$ and $\epsilon$ denoting the non-empty set and sufficiently small positive constant, respectively. Then, the following result is obtained; that is, the neighborhood set $N_{i}$ of agent $i$ is defined as the set of agents (or nodes) that are adjacent to agent $i$, that is $N_{i}=\{(i, j) \in E, E \in(V, V)\}$.

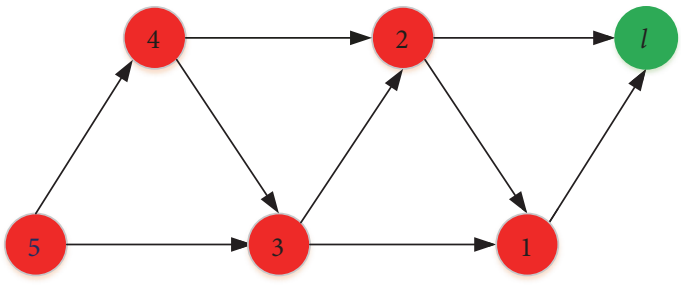

FIGURE 1: Information interaction of network under the leader follower structure. The blue circle and red circles denote the leader $l$ and followers, labelled by $1, \ldots, 5$, respectively.

Figure 1 shows the information topology $G$ for directed graph of six nodes (viewed as agents). As per the directed graph theory, the following results are directly obtained as follows: $V=\{l, 1,2,3,4,5\}, E=$ $\{(1, l),(2, l),(2,1),(3,1),(3,2),(4,2),(4,3),(5,3),(5,4)\}$.

Assume that the total neighbor set for six agents, i.e., denoted by $N_{(G)}=N_{l} \cup N_{1} \cup N_{2} \cup N_{3} \cup N_{4} \cup N_{5}$ with $N_{l}=\{\emptyset\}, N_{1}=\{l\}$, $N_{2}=\{1\}, N_{3}=\{1,2\}, N_{4}=\{2,3\}$, and $N_{5}=\{3,4\}$, respectively. There is no loop, i.e., $m_{i i}=0$ and self-loop in the directed graph.

Assumption 1. To generate the sensing interaction topology $G$ of the directed graph, e.g., as shown in Figure 1, there exist any three edges $e_{i j}, e_{j k}$, and $e_{k i}$ in the edge set $E$ such that the directed graph satisfies the following conditions: $\left\|e_{i j}\right\|+$ $\left\|e_{j k}\right\|<\left\|e_{k i}\right\|,\left\|e_{i j}\right\|+\left\|e_{k i}\right\|<\left\|e_{j k}\right\|$, and $\left\|e_{j k}\right\|+\left\|e_{k i}\right\|<\left\|e_{i j}\right\|$. The connected graph considered in this study is assumed.

We further assume that the desired (or target) formation is infinitesimally rigid by applying the Henneberg sequence in this study $[10,26]$.

Assumption 2. The control gain $\gamma_{i j}(\cdot)$ of agent $i$ is continuous and Lipschitz, which only depends on the difference $y_{i j}$ of inter-distance between all pairs of agents, for all $(i, j) \in E$, namely, $\gamma_{i j}\left(\alpha_{i j}, y_{i j}\right)=s_{i j} \alpha_{i j}\left(\exp \left(y_{i j}\right)-1\right)$ for $y$; otherwise, $\gamma_{i j}\left(\alpha_{i j}\right.$, $\left.y_{i j}\right)=s_{i j} y_{i j}$, where $\alpha_{i j} \in R^{+}$are hyper-parameters, and $\gamma_{i j} \in R^{+}$ are scale factors. Note that $\alpha_{i j}$ is also interpreted as interaction function.

Remark 3. To obtain the control law for each follower agent $i$, we introduce the nonlinear control gain $\gamma_{i j}\left(\alpha_{i j}, y_{i j}\right)$. As expected, agent $i$ can follow its neighbors with the same direction (or orientation), which can guarantee the desired convergence performance for the overall system under the Assumption 2. In other words, $y_{i j}=0$ implies $\gamma_{i j}\left(\alpha_{i j}, y_{i j}\right)=$ 0 , the desired formation is achieved, as will be shown in Section 4. Note that the distributed control law of agent $i$ for the part of controlling the geometrical formation is nonlinear.

To solve the obstacle avoidance problem, we introduce the concept of artificial potential function as follows.

The artificial potential function (APF) $V_{A P F}\left(y_{i k}\right)$ is a nonnegative differentiable and radially unbounded between agents $i$ and obstacles $k$ such that

(a) $V_{A P F}\left(y_{i k}\right) \longrightarrow 0$ if and only if $y_{i k} \geq R_{A P F}$,

(b) $V_{A P F}\left(y_{i k}\right) \longrightarrow \infty$ as $y_{i k}$ approaches to zero, 
where $y_{i k}$ denotes the distance errors between the actual distance $d_{i k}$ and the minimal repulsive distance threshold $\delta_{i k}$, i.e., $y_{i k}=\left|d_{i k}-\delta_{i k}\right|, R_{A P F}$ denotes the maximal distance that the artificial potential can be generated. It is further assumed that $R_{A P F}$ is less than the sensing range $R_{s}$ of each agent, i.e., $R_{A P F}<R_{s}$.

Consider an artificial potential function (APF) for agents $i$ as

$$
V_{i \_A P F}=\sum_{k=1}^{m} V_{A P F}\left(y_{i k}\right)
$$

The time derivative of $V_{i_{-} A P F}\left(y_{i k}\right)$ is given as

$$
\dot{V}_{i \_A P F}=\nabla_{q_{i}} \sum_{k=1}^{m} V_{A P F}\left(y_{i k}\right)
$$

Applying the gradient-based control approach, the repulsive force for each agent $i$ is defined as

$$
u_{o b s}=-\dot{V}_{i_{-} A P F}
$$

If agent is approaching the obstacle $k$, the repulsive force is generated to steer agent $i$ to move toward gradient direction of the repulsive potential function (3), i.e., the agent $i$ is driven away from the obstacle, which implies that obstacle avoidance problem can be solved by implementing the obstacle control law $u_{o b s}$, implying that the agents can be steered away from any possible encountered obstacle.

\section{Problem Statement and Control Objective}

3.1. System Model. It is assumed that each agent is endowed with second-order integrator dynamics. Therefore, the multiagent system consisting of $n$ agents is characterized as

$$
\begin{aligned}
& \dot{q}_{i}=p_{i}, \\
& \dot{p}_{i}=f\left(q_{i}, p_{i}\right)+g\left(q_{i}, p_{i}\right) u_{i}
\end{aligned}
$$

where $q_{i} \in R^{m}$ is the position and $p_{i} \in R^{m}$ is the velocity. The position and control input of the agents can be stacked into $q=\left[q_{1}^{T}, \ldots, q_{i}^{T}, \ldots, q_{n}^{T}\right]^{T}$ with $q_{i}=\left[q_{i 1}, \ldots, q_{i m}\right]^{T} \in R^{m}$, and $p=\left[p_{1}^{T}, \ldots, p_{i}^{T}, \ldots, p_{n}^{T}\right]^{T}$ with $p_{i}=\left[p_{i 1}, \ldots, p_{i m}\right]^{T} \in R^{m}$, respectively. $u_{i}=\left[u_{i 1}, \ldots, u_{i m}\right]^{T} \in R^{m}$ is the control input, and $u=\left[u_{1}, \ldots, u_{m}\right] . f(\cdot) \in R^{m}$ is the continuously differentiable function with $f(\cdot)=0 ; g(\cdot) \in R^{m}$ are the smooth functions with $g(\cdot) \neq 0$ for all $q$ and $p$.

To continue the analysis, we define the relative position and their error vectors as

$$
\begin{aligned}
& z_{i r}=q_{i}-q_{r}, \\
& z_{i j}=q_{i}-q_{j} .
\end{aligned}
$$

and

$$
\begin{array}{r}
e_{q i r}=z_{i r}-z_{i r}^{d}, \\
e_{p i r}=p_{i}-p_{r} . \\
\text { and } e_{q i j}=z_{i j}-z_{i j}^{d}, \\
e_{p i j}=p_{i}-p_{j} .
\end{array}
$$

respectively.
Taking the first derivative of (8a) and (8b), we obtain the error dynamics

$$
\begin{aligned}
\dot{e}_{q i r} & =e_{p i r}, \\
\dot{e}_{p i r} & =\dot{p}_{i}-\dot{p}_{r} \\
\text { and } \dot{e}_{q i j} & =e_{p i j}, \\
\dot{e}_{p i j} & =\dot{p}_{i}-\dot{p}_{j}
\end{aligned}
$$

As discussed in the above sections, it is assumed that the leader dynamics is given as

$$
\begin{aligned}
& \dot{q}_{r}=p_{r}, \\
& \dot{p}_{r}=f\left(q_{r}, p_{r}\right)
\end{aligned}
$$

where $q_{r} \in R^{m}$ and $p_{r} \in R^{m}$ represent the position and velocity parameters of leader agent, respectively. Note that the knowledge of (10) is now available to the follower agents.

Remark 4. Under the leader following control scheme, equation (6) is used to characterize the reference trajectory for a team of agents. Then, the desired formation can be achieved and maintained under the manipulation of the control law proposed in the sequel.

Assumption 5 (see $[11,25])$. A function $f(\cdot)$ with domains $P$ and $Q$ is Lipschitz if there exist two positive constants $\zeta_{L}=$ $\left[\zeta_{L q}, \zeta_{L p}\right] \in R^{+}:$for $p \in P, q \in Q$, and $i=1, \ldots, n$.

$$
\|\tilde{f}(\cdot)\| \leq \zeta_{L q}\left\|q_{i}-q_{r}\right\|+\zeta_{L p}\left\|p_{i}-p_{r}\right\|
$$

with

$$
\tilde{f}(\cdot)=f\left(q_{i}, p_{i}\right)-f\left(q_{r}, p_{r}\right)
$$

Note that $\zeta_{L}=\left[\zeta_{L q}, \zeta_{L p}\right]$ are viewed as the Lipschitz operators.

Assumption 6. We assume that two system reference variables $q_{r}$ and $p_{r}$ for the leader agent satisfy the following properties:

$$
\begin{aligned}
& -\varsigma_{1} \leq q_{r} \leq \varsigma_{1}, \\
& -\varsigma_{2} \leq p_{r} \leq \varsigma_{2} .
\end{aligned}
$$

for some positive constants $\varsigma_{1} \in R^{+}$and $\varsigma_{2} \in R^{+}$.

Assumption 7 (see [18]). For a given desired formation control, a Lyapunov function candidate $V(z)$ and $z(p)$ satisfy the following constraints.

(a) $\Theta(\gamma)$ is compact for any $\gamma \geq 0$.

(b) There exist $\gamma_{e}=\left[\gamma_{e p}, \gamma_{e q}\right]>0$ such that $V(z)=0$ if and only if $\|z\|=\gamma_{e}$ in $\Theta(\gamma)$. Note that the converse is also true, i.e., $\|z\|=\gamma_{e}$ implies $V(z)=0$.

(c) For any given bounded $\|z\|,\|\partial z(p) / \partial p\|$ and $\|u(z, p)\|$ are bounded.

Assumption 7 means that $\|z\|=\gamma_{e}$ are the asymptotically stable equilibrium values for the system. $\Theta(\gamma)$ is viewed as the attraction region owing to the invariance set principle. The 
formation control system is locally stable if $\Theta(\gamma)$ is the local space; the system is globally stable, otherwise. In practice, it is difficult to obtain the attraction region of the whole space for nonlinear control systems. It is noted that the constraint (b) in Assumption 7 implies Assumption 6. For example, in distance-based formation control, as addressed in [8, 10, 27], the desired formation can be achieved if and only if the actual interdistance converges to the desired interdistance as time tends to infinity. Thus, Assumption 7 is reasonable and verified.

With the above results at hand, we introduce the following useful definition.

Definition 8 (achieving the desired formation). The desired formation for a multiagent is said to be achieved if the following constraints are satisfied under the Assumptions 5 -7 .

$$
\begin{aligned}
& \lim _{t \rightarrow \infty}\left(q_{i}-q_{r}\right)=q_{i}^{d}-q_{r}^{d} \\
& \lim _{t \rightarrow \infty}\left(q_{i}-q_{j}\right)=q_{i}^{d}-q_{j}^{d}, \quad j \in N_{i}
\end{aligned}
$$

and

$$
\begin{aligned}
& \lim _{t \rightarrow \infty}\left(p_{i}-p_{r}\right)=0, \\
& \lim _{t \rightarrow \infty}\left(p_{i}-p_{j}\right)=0, \quad j \in N_{i} .
\end{aligned}
$$

respectively. Here, $q_{i}^{d}-q_{r}^{d}$ is the desired local displacement between agent $i$ and the leader, and $q_{i}^{d}-q_{j}^{d}$ is the desired local displacement (or position) between agent $i$ and follower agent $j$. The velocity $p_{i}$ of agent $i$ converges to the reference velocity $p_{r}$ as time $t$ evolves. Note that equation (9a) and (9b) not only determines the orientation of the desired formation but also specifies the desired interdistances among agents by calculating the magnitudes of $q_{i}^{d}-q_{r}^{d}$ and $q_{i}^{d}-q_{j}^{d}$, respectively.

We are now prepared to state the control objective for multiagent system as follows.

3.2. Control Objective. The control objective in this study is to design the distributed control law such that the system satisfies the physical constraints given as follows:

(a) achieve the desired formation shape;

(b) track the reference trajectory with the same reference velocity for each follower agent;

(c) solve the obstacle avoidance problem.

To fulfill the control objective, we first present the error dynamics as follows.

Substituting (6), (10), and (12) into (9a) and (9b), yields

$$
\begin{aligned}
& \dot{e}_{q i r}=e_{p i r}, \\
& \dot{e}_{p i r}=\tilde{f}(\cdot)+g\left(q_{i}, p_{i}\right) u_{i r} .
\end{aligned}
$$

which is expressed in a condensed form as

$$
\begin{aligned}
{\left[\begin{array}{c}
\dot{e}_{\text {qir }} \\
\dot{e}_{\text {pir }}
\end{array}\right]=} & {\left[\begin{array}{cc}
0_{m \times m} & I_{m} \\
0_{m \times m} & 0_{m \times m}
\end{array}\right]\left[\begin{array}{c}
e_{q i r} \\
e_{\text {pir }}
\end{array}\right]+\left[\begin{array}{c}
0_{n \times m} \\
\tilde{f}(\cdot)
\end{array}\right] } \\
& +\left[\begin{array}{c}
0_{n \times m} \\
g\left(q_{i}, p_{i}\right) u_{i r}
\end{array}\right]
\end{aligned}
$$

and

$$
\begin{aligned}
& \dot{e}_{p i j}=e_{q i j}, \\
& \dot{e}_{q i j}=\tilde{f}(\cdot)+g\left(q_{i}, p_{i}\right) u_{i j} .
\end{aligned}
$$

which is also rewritten in a condensed form as

$$
\begin{aligned}
{\left[\begin{array}{c}
\dot{e}_{q i j} \\
\dot{e}_{p i j}
\end{array}\right]=} & {\left[\begin{array}{cc}
0_{m \times m} & I_{m} \\
0_{m \times m} & 0_{m \times m}
\end{array}\right]\left[\begin{array}{l}
e_{q i j} \\
e_{p i j}
\end{array}\right]+\left[\begin{array}{c}
0_{n \times m} \\
\tilde{f}(\cdot)
\end{array}\right] } \\
& +\left[\begin{array}{c}
0_{n \times m} \\
g\left(q_{i}, p_{i}\right) u_{i j}
\end{array}\right]
\end{aligned}
$$

respectively, where $e=\left[e_{q}^{T}, e_{p}^{T}\right]^{T}, e_{q}^{T}=\left[e_{q 1}^{T}, \ldots, e_{q n}^{T}\right]^{T}$, $e_{p}^{T}=\left[e_{p 1}^{T}, \ldots, e_{p n}^{T}\right]^{T}, f(\cdot)=\left[f_{1}^{T}(\cdot), \ldots, f_{n}^{T}(\cdot)\right]^{T}$, and $u(\cdot)=$ $\left[u_{1}^{T}, \ldots, u_{n}^{T}\right]^{T}$.

\section{Formation Control and Obstacle Avoidance}

As discussed in the previous sections, it is supposed that each follower has no more than two neighbors in the information interaction topology of the directed graph. The leader has access to all the information, i.e., position and velocity, of the reference trajectory, while the followers do not know the information from the reference trajectory. To achieve the control target, we propose the control law as follows, the stability analysis of multiagent system is provided, then the distributed control algorithm for obstacle avoidance problem is presented. As a result, we analyze the stability properties of system according to the formation and obstacle control, without loss of generality, followed by the associated proofs.

4.1. Design of Formation Control. The general formation control law $u_{i}$ is given as

$$
\begin{gathered}
u_{i}=\frac{1}{g\left(q_{i}, p_{i}\right)}(\underbrace{u_{i j}+u_{i r}}_{\text {Formation control term }} \\
+\underbrace{\left.u_{\text {a_obs }}-\tilde{f}(\cdot)\right)}_{\text {obstacle avoidance term }}
\end{gathered}
$$

where

$$
\begin{aligned}
& u_{i j}=m_{i j}\left(q_{j}-q_{i}+q_{i}^{d}-q_{j}^{d}\right)+m_{i j}\left(p_{j}-p_{i}\right), \\
& u_{i r}=m_{i k}\left(q_{j}-q_{i}+q_{i}^{d}\right)+m_{i k}\left(p_{j}-p_{i}\right) .
\end{aligned}
$$


with $\left\|q_{i}^{d}-q_{i}^{d}\right\|=d_{i j}^{d} \in R^{+},\left\|q_{i}^{d}\right\|=d_{i r}^{d} \in R^{+}$, and $\left\|q_{j}^{d}\right\|=$ $d_{j r}^{d} \in R^{+}$denoting the desired distance of edges which connect agent $i$ to $j$ and the leaders $l$, respectively, which satisfy Assumptions 1 and 2. The control in (20) is clearly distributed as the control of agent $i$ only relies on the local measurements from its neighboring agents (the leader or other followers). Here, $m_{i j} \in R$ and $m_{i r} \in R$ denote some constant numbers, respectively; they are taken from the adjacency matrix $M$. Physically, $m_{i j}$ represents the interaction weights between agents $i$ and $j$, and $m_{i k}$ represents the interaction weights between agent $i$ and $k$-th leader agent. $u_{i_{\text {_obs }}}$ will be given in Section 4.3. As observed in (21), the control gains are some unknown positive constants; therefore, equation (21) is clearly linear, while in many practical applications, the control gains (or called weights) are varied with the interdistances between the follower agent $i$ and their neighbors. To better characterize these behaviors, mathematically speaking, we will explicitly give the notion of $\gamma_{i j}\left(\alpha_{i j}, y_{i j}\right)$ and $\gamma_{i r}\left(\alpha_{i r}, y_{i r}\right)$ as follows.

The control gains $\gamma_{i j}\left(\alpha_{i j}, y_{i j}\right)$ and $\gamma_{i r}\left(\alpha_{i r}, y_{i r}\right)$ [11] for the follower agent $i$ and the leaders and follower agent $j$ are assumed as

$$
\gamma_{i r}\left(\alpha_{i r}, y_{i r}\right)=s_{i r} \begin{cases}\frac{1}{1+\exp \left(y_{i r}\right)}, & y_{i r}<0 \\ d_{i r}, & \text { otherwise. }\end{cases}
$$

and

$$
\gamma_{i j}\left(\alpha_{i j}, y_{i j}\right)=s_{i j} \begin{cases}\frac{1}{1+\exp \left(y_{i j}\right)}, & \mathrm{y}_{i j}<0, \\ d_{i j}, & \text { otherwise. }\end{cases}
$$

respectively.

Here, $s_{i j}$ and $\alpha_{i j}$ remain the same as Assumption 2. $y_{i j}$ is defined as $y_{i j}=\left\|z_{i j}\right\|-\left\|z_{i j}^{d}\right\|=d_{i j}-d_{i j}^{d}$, for all $i, j=\{m-1, m-$ $2, \ldots, n\}$.

Consider the follower agent and the leaders, for ease of notation, simply called $j$. As a result, the geometrical interpretation of (18) is provided and stated as follows: (1) agent $i$ moves away from the agent $j$ if the initial distance $\left\|z_{i j}\right\|$ is less than the desired distance $\left\|z_{i j}^{d}\right\|$, and then the control gains are chosen as $s_{i j} /\left(\exp \left(y_{i j}\right)+1\right) ;(2)$ agent $i$ moves toward from the agent $j$ if the initial distance $\left\|z_{i j}\right\|$ is greater than the desired distance $\left\|z_{i j}^{d}\right\|$, and then the control gains are selected as $s_{i j} d_{i j}$. Therefore, the desired formation is achieved if and only if $y_{i j}=0$ as time $t$ evolves, i.e., $\left\|z_{i j}\right\|=\left\|z_{i j}^{d}\right\|$, which implies the actual velocity $v_{i}$ converges to the reference velocity $v_{r}$ in terms of (7), (8a), and (8b).

As outlined in the previous sections, in order to achieve the control objective, assume that at least one follower has a neighbor of the leader agent. By replacing $m_{i j}$ and $m_{i k}$ with $\gamma_{i j}\left(\alpha_{i j}, y_{i j}\right)$ and $\gamma_{i k}\left(\alpha_{i k}, y_{i k}\right)$, respectively, in terms of (8a) and $(8 \mathrm{~b})$, we obtain

$$
\begin{aligned}
e_{q i}(\cdot)= & m_{i j} \cdot \gamma_{i j}\left(\alpha_{i j}, y_{i j}\right)\left(q_{j}-q_{i}+q_{i}^{d}-q_{j}^{d}\right)+m_{i j} \\
& \cdot \gamma_{i j}\left(\alpha_{i j}, y_{i j}\right)\left(q_{k}-q_{i}+q_{i}^{d}\right)
\end{aligned}
$$

and

$$
\begin{aligned}
e_{p i}(\cdot)= & m_{i j} \cdot \gamma_{i j}\left(\alpha_{i j}, y_{i j}\right)\left(p_{j}-p_{i}\right)+m_{i j} \\
& \cdot \gamma_{i j}\left(\alpha_{i j}, y_{i j}\right)\left(p_{k}-p_{i}\right)
\end{aligned}
$$

respectively.

Differentiating (24) and (25), we have

$$
\begin{gathered}
\dot{e}_{q i}(\cdot)=m_{i j} \cdot \gamma_{i j}\left(\alpha_{i j}, y_{i j}\right)\left(p_{j}-p_{i}\right)+m_{i j} \\
\cdot \gamma_{i j}\left(\alpha_{i j}, y_{i j}\right)\left(p_{k}-p_{i}\right)=e_{p i}
\end{gathered}
$$

and

$$
\dot{e}_{p i}(\cdot)=u_{i}+\widetilde{f}(\cdot)
$$

respectively.

To continue the analysis, define the formation control as

$$
u_{i}=\frac{1}{g\left(q_{i}, p_{i}\right)}\left(e_{q i}+e_{p i}+u_{i_{-o b s}}-\tilde{f}(\cdot)\right)
$$

It is noted that (28) represents distributed (or decentralized) nonlinear control laws as (28) only depends on the local information from their neighbors.

Substituting (28) into (27) yields

$$
\dot{e}_{p i}(\cdot)=e_{q i}+e_{p i}+u_{i_{\_} o b s}
$$

With the above results in hand, we are now ready to present the formation stability analysis as follows. As a rule, the stability analysis for the overall system is divided into two parts: (1) formation stability analysis and (2) obstacle avoidance analysis. The analyses for the above two parts are presented in Sections 4.2 and 4.3, respectively, as follows.

4.2. Formation Stability Analysis. Define the Lyapunov function candidate as

$$
V_{i F}=\frac{1}{2} \sum_{i=1}^{n}\left(e_{q i}^{2}+e_{p i}^{2}\right)
$$

which is continuously differentiable.

To proceed, we define the formation error dynamics as

$$
\left[\begin{array}{c}
\dot{e}_{q i} \\
\dot{e}_{p i}
\end{array}\right]=-(\gamma_{i}(\cdot) \underbrace{\left(\begin{array}{cc}
0_{n \times n} & -I_{n} \\
L+\Delta_{L} & L+\Delta_{L}
\end{array}\right)}_{A_{L}} \otimes I_{m})\left[\begin{array}{c}
e_{q i} \\
e_{p i}
\end{array}\right]
$$

where $\gamma_{i}(\cdot)=\operatorname{diag}\left\{\gamma_{i k}, \ldots, \gamma_{i j}, \ldots, \gamma_{i n}\right\}$.

Taking the first time derivative of $V_{i F}$, one gets

$$
\begin{aligned}
\dot{V}_{i F} & =e_{q i}^{T} \bar{Q} \dot{e}_{q i}+e_{p i}^{T} \bar{Q} \dot{e}_{p i} \\
& =-\left(e_{q i}^{T} \bar{Q} e_{p i}+e_{p i}^{T} \bar{Q}\left(e_{q i}+e_{p i}\right)\right)
\end{aligned}
$$

Substituting (31) into (32) yields

$$
\dot{V}_{i F}=-\left[\begin{array}{c}
e_{q i} \\
e_{p i}
\end{array}\right]^{T} \mathbb{Q}\left[\begin{array}{l}
e_{q i} \\
e_{p i}
\end{array}\right] \leq 0
$$


where

$$
\begin{aligned}
\mathbb{Q} & =\gamma_{i}(\cdot)\left(\left(\left(\begin{array}{cc}
0_{n \times n} & I_{n} \\
L+\Delta_{L} & L+\Delta_{L}
\end{array}\right) \otimes I_{m}\right)^{T} \bar{Q}\right. \\
& \left.+\bar{Q}^{T}\left(\left(\begin{array}{cc}
0_{n \times n} & I_{n} \\
L+\Delta_{L} & L+\Delta_{L}
\end{array}\right) \otimes I_{m}\right)\right)
\end{aligned}
$$

Since the positive semidefinite of (34), it follows that (33) is verified.

Theorem 9. Consider the multiagent system (6) with the reference trajectory (10) under the leader-following structure. For any given bounded initial condition, the control objective is achieved under the action of the proposed control law (20).

Proof. The Lyapunov function candidate in (30) attains the minimal value if and only if $e_{q i}$ and $e_{p i}$ are simultaneously equal to zero, i.e., $z_{i j}-z_{i j}^{d}=0, z_{i k}-q_{i}^{d}=0$, and $p_{i}=$ $p_{j}=p_{r}$ because of nonnegative control gains $\gamma_{i j}\left(\alpha_{i j}, y_{i j}\right)$ and $\gamma_{i k}\left(\alpha_{i k}, y_{i k}\right)$. In terms of (33), the first time derivative of $V_{i F}$ is seminegative positive, i.e., $\partial V_{i F} / \partial t \leq 0$, implying that $V_{i F}(\mathrm{t})$ $-V_{i F}(0) \leq 0$. Therefore, the system is asymptotically stable. This concludes the proof.

Lemma 10. The matrix $A_{L}$ in (31) is positive definite, implying that $-A_{L}$ is Hurwitz (or stability), for any $m_{i j}$ and $s_{i j}$; namely, the system is asymptotically stable.

Proof. Letting $\lambda$ be the eigenvalues of $-A_{L}$, then

$$
\begin{aligned}
\operatorname{det} & \left(\lambda I_{n}+A_{L}\right) \\
= & \operatorname{det}\left(\begin{array}{cc}
\lambda I_{n} & -\gamma_{i}(\cdot) \\
\gamma_{i}(\cdot)\left(L+\Delta_{L}\right) & \lambda I_{n}+\gamma_{i}(\cdot)\left(L+\Delta_{L}\right)
\end{array}\right) \\
= & \operatorname{det}\left(\lambda^{2} I_{n}+\lambda \gamma_{i}(\cdot)\left(L+\Delta_{L}\right)+\gamma_{i}^{2}(\cdot)\left(L+\Delta_{L}\right)\right)
\end{aligned}
$$

As a consequence, $\operatorname{det}\left(\lambda I_{n}+A_{L}\right)=0$, and we obtain

$$
\begin{aligned}
\lambda= & \frac{1}{2}\left(-\gamma_{i}(\cdot)\left(L+\Delta_{L}\right)\right. \\
& \left.+\gamma_{i}(\cdot) \sqrt{\left(L+\Delta_{L}\right)^{2}-4\left(L+\Delta_{L}\right)}\right)
\end{aligned}
$$

The eigenvalues $\lambda$ in (36) have a strictly negative real part according to Definition 8, implying that $-A_{L}$ is Hurwitz, which completes the proof.

4.3. Obstacle Avoidance in the Leader-Follower Structure. In this subsection, we propose a distributed control strategy for avoiding obstacles of a group of autonomous agents based on the leader-follower structure. In this study, geometrically, it is assumed that each obstacle $\mathrm{k}$ is viewed as a circle. Without loss of generality, the boundary of each obstacle can be recognized by each agent equipped with visual sensor.
Consider the artificial potential function as

$$
V_{i \_A P F}= \begin{cases}\sum_{k=1}^{m}\left(d_{i k}-\delta_{i k}\right)^{-2}, & \delta_{i k}<d_{i k} \leq R_{A P F} . \\ 0, & d_{i k}>R_{A P F} .\end{cases}
$$

where $d_{i k}=\left\|q_{i}-q_{k}\right\|$ denotes the distance between agent $i$ and obstacle $k$. $\delta_{i k}$ remains the same as in Definition 8.

From (37), the artificial potential function is unbounded when agent $i$ gets close to the obstacle $k$, namely, $d_{i k} \longrightarrow \delta_{i k}$. The artificial potential is produced or vanishes if the distance $d_{i k}$ lies in $\left(\delta_{i k}, R_{A R F}\right)$ or $d_{i k}>R_{A R F}$. As a result, the associated repulsive force for each agent $i$ is devised as

$$
\begin{aligned}
& u_{i-o b s} \\
& = \begin{cases}0, & d_{i k}>R_{A P F} . \\
-\sum_{k=1}^{m} 2\left(d_{i k}-\delta_{i k}\right)^{-3} \frac{\left(p_{i}-p_{j}\right)}{d_{i k}}, & \delta_{i k}<d_{i k} \leq R_{A P F} .\end{cases}
\end{aligned}
$$

Using the control law (38), we can solve the obstacle avoidance problem.

Agent 1 can avoid two obstacles without getting stuck in the local minimum points by using the attractive force produced by agent 1 and the reference trajectory, which is illustrated in Figure 2.

4.4. Bounded Constant and Time-Varying Disturbances. In many practical applications, multiagent systems are vulnerable to exterior constant disturbances. The constant disturbances (which can sometimes be viewed as constant velocities) cannot be rejected by the distributed proportional control laws. Toward this end, we apply the PI controller for double-integrator dynamics to achieve the desired formation under the constant (or static) disturbances.

To continue our analysis, we analyze a distributed (or decentralized) PI formation controller with the constant disturbances for a multiagent system, where each agent $i$ only uses the local position and velocity measurements from its neighbors. Hence, this controller not only provides an advantage of eliminating the static errors but also improves the convergence performance. Therefore, the desired formation is achieved under the proposed control laws in the presence of constant disturbances. We first devise the distributed controller for agent $i$ as

$$
\begin{aligned}
& \dot{\xi}_{i j}=q_{j}-q_{i}+q_{i}^{d}-q_{j}^{d}, \\
& u_{i}=m_{i k}\left(q_{j}-q_{i}+q_{i}^{d}\right)+m_{i k}\left(p_{j}-p_{i}\right)+c_{i} \xi_{i j} .
\end{aligned}
$$

with $c_{i}$ denoting some positive constant number, which is referred to as integral gains. Equation (39) relies only on the local measurements. The diagram of the distributed PI controller is shown in Figure 3. Note that the system for each agent $i$ may be unstable when a minor local measurement error occurs.

Integrating the first equation of (39), we obtain

$$
\xi_{i j}=\left(q_{i}^{d}-q_{j}^{d}\right) t-\int_{0}^{t}\left(q_{i}(\tau)-q_{j}(\tau)\right) d \tau
$$




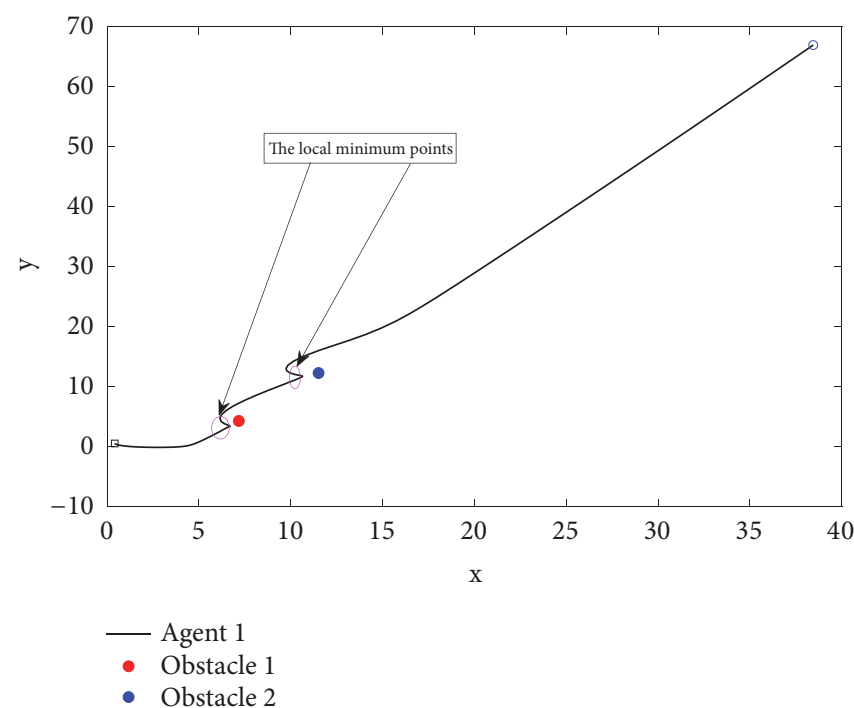

FIGURE 2: Obstacle avoidance of agent 1. The blue circle and black square denote the initial and final positions of agent 1, respectively. Two different obstacles are considered, which are colored with red and blue, respectively. The local minimum points are given.

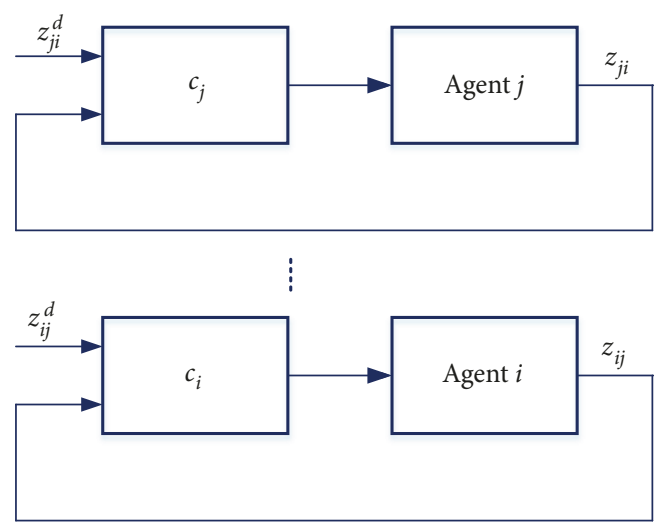

FIGURE 3: Block diagram of distributed PI controller. Agent $i$ only has access to the local measurements.

From (36), each agent $i$ has only access to local relative positions (or measurements) and the time for obtaining the desired estimate of the integral error states.

Consider again the double-integrator dynamics for multiagent systems as

$$
\begin{aligned}
& \dot{q}_{i}=p_{i} \\
& \dot{p}_{i}=u_{i}+d_{i}
\end{aligned}
$$

with $d_{i}$ denoting the unknown constant disturbances.

The main result of this part is given by Theorem 11 as follows.

Theorem 11. Consider the multiagent system (41) under the connected interaction graph $G$. Using the formation control law (39), the static errors are eliminated, and the desired formation is achieved under the Assumption 4.1, i.e., $\lim _{t \rightarrow \infty} q_{i}-q_{j}=q_{d i}$ $-q_{d j}$ and $\lim _{t \rightarrow \infty} p_{i}-p_{j}=0$ for $i=1 \ldots, n, j \in N_{i}$.

Remark 12. To address the problem in the presence of the constant disturbances, we introduce the control term of integral action, which can eliminate the static errors and guarantee the desired performance. In other words, the actual relative position vector converges to the specified relative position vector; thus, the control objective is achieved.

Proof. From (39) and (41), by introducing a new state vector $\xi_{i}=\left[\xi_{1 j}, \ldots, \xi_{n j}\right]^{T}$, we may write the corresponding augmented matrix in a compact form as

$$
\begin{aligned}
& {\left[\begin{array}{c}
\dot{\xi}_{i} \\
\dot{e}_{q i} \\
\dot{e}_{p i}
\end{array}\right]} \\
& =\left[\begin{array}{ccc}
0_{n \times n} & I_{n} & 0_{n \times n} \\
0_{n \times n} & 0_{n \times n} & I_{n} \\
-k_{q i}\left(L+\Delta_{L}\right) & -k_{p i}\left(L+\Delta_{L}\right) & -c_{i} I_{n}
\end{array}\right]\left[\begin{array}{c}
\xi_{i} \\
e_{q i} \\
e_{p i}
\end{array}\right] \\
& +\left[\begin{array}{c}
0_{n \times 1} \\
0_{n \times 1} \\
c_{n \times 1}
\end{array}\right]\left(q_{i}^{d}-q_{j}^{d}\right)+\left[\begin{array}{c}
0_{n \times 1} \\
0_{n \times 1} \\
d_{n \times 1}
\end{array}\right]
\end{aligned}
$$

for $i=\{1, \ldots, n\}$, together with the bounded initial condition. Here, $k_{q i} \in R^{+}$and $k_{p i} \in R^{+}$are positive numbers, respectively, $q_{i}^{d}-q_{j}^{d}$ is the desired relative position vector given in advance, and $d_{n \times 1}$ are constant (static) disturbances. Note that the aim of (42) is to stabilize system with respect to the parameters, i.e., $\xi_{i}, e_{q i}, e_{p i}$. 
Taking the time derivative of (42), we obtain

$$
\begin{aligned}
& {\left[\begin{array}{c}
\ddot{\xi}_{i j} \\
\ddot{e}_{q i} \\
\ddot{e}_{p i}
\end{array}\right]} \\
& =\underbrace{\left[\begin{array}{ccc}
0_{n \times n} & I_{n} & 0_{n \times n} \\
0_{n \times n} & 0_{n \times n} & I_{n} \\
-k_{q i}\left(L+\Delta_{L}\right) & -k_{p i}\left(L+\Delta_{L}\right) & -c_{i} I_{n}
\end{array}\right]}_{:=A_{A u g}}\left[\begin{array}{c}
\dot{\xi}_{i j} \\
\dot{e}_{q i} \\
\dot{e}_{p i}
\end{array}\right]
\end{aligned}
$$

To obtain the eigenvalues of $A_{A u g}$, the characteristic polynomial of $A_{A u g}$ is calculated as

$$
\begin{aligned}
\operatorname{det}\left(A_{A u g}\right)= & \left|\lambda I_{n}-A_{A u g}\right| \\
= & \left|\begin{array}{ccc}
s I_{n} & -I_{n} & 0_{n \times n} \\
-0_{n \times n} & s I_{n} & -I_{n} \\
k_{q i}\left(L+\Delta_{L}\right) & k_{p i}\left(L+\Delta_{L}\right) & s I_{n}+c_{i} I_{n}
\end{array}\right| \\
= & s^{3}+c_{i} s^{2}+k_{p i}\left(L+\Delta_{L}\right) s \\
& +k_{q i}\left(L+\Delta_{L}\right)=0
\end{aligned}
$$

To continue the analysis, consider the characteristic polynomial of $\left(L+\Delta_{L}\right)$ as

$$
s^{3}+c_{i} s^{2}+k_{p i} \lambda_{i} s+k_{q i} \lambda_{i}=0
$$

where $\lambda_{i} \in R^{+}$are the eigenvalues of $\left(L+\Delta_{L}\right)$, i.e., $\varsigma_{i}>0$.

Comparing (44) with (45), we learn that all the eigenvalues of $A_{A u g}$ have strictly negative real parts because of the positive eigenvalues of $\lambda_{i}$ as mentioned before, which implies $A_{A u g}$ is Hurwitz (or stable), and the system is therefore asymptotically stable. Furthermore, one can also place the poles according to the characteristic polynomial (47) such that the system is asymptotically stable. To this end, the control gain can be easily computed by using the command of place (.) in MATLAB. As discussed, $A_{A u g}$ is Hurwitz, and we then obtain

$$
s^{3}+c_{i} s^{2}+k_{p i} \lambda_{i} s+k_{q i} \lambda_{i}=0
$$

From (46), one can get

$$
\lim _{t \rightarrow \infty} q_{j}-q_{i}=q_{i}^{d}-q_{j}^{d}
$$

which implies that the proposed distributed PI controller (39) can eliminate the static errors caused by constant but unknown disturbances and implies that the relative positions between agents $i$ and $j$ converge to the desired relative positions, the desired formation is achieved and, maintained, meanwhile the velocity errors between agents $i$ and $j$ converge to zero. The proof is thus completed.

Remark 13. If the errors are equal to zero, i.e., the actual relative position equals the desired relative position, the proposed controller stops to calculate the changes, implying that the desired formation is achieved. It is noted that the convergence rate of the overall system is dominated by the smallest eigenvalue of $A_{\text {Aug }}$.

Example 14. Without loss of generality and for brevity, consider an agent 1 with initial position $q_{1}(0)=\left[\begin{array}{ll}0.42 & 0.50\end{array}\right]^{T}$ and its second-order dynamics (41) with the bounded constant disturbances given as $d_{1}=\left[\begin{array}{ll}0.78 & 0.25\end{array}\right]^{T}$ and $d_{2}=$ $\left[\begin{array}{ll}3.26 & 4.92\end{array}\right]^{T}$, and the control gains are $k_{q}=1.35$ and $k_{p}=1.35$ and integral gain $c_{i}=0.75$, respectively.

Figure 4 illustrates the trajectories of agent 1 in the presence of disturbances, where the left and right figures show the comparison results concerning the static performance between with and without the aim of integral control terms. As expected, the static error is successfully eliminated by introducing the integral control terms, depicted by blue lines in the left subfigures, whereas the static error is not eliminated without the aim of the integral control, shown in red lines in the right subfigures, in $\mathrm{x}$ and $\mathrm{y}$ coordinates, respectively. The right two subfigures show the convergence performance of the system in the presence of two different constant disturbances. The convergence rate is bigger when the disturbance is smaller, i.e., $d_{1}=\left[\begin{array}{ll}0.78 & 0.25\end{array}\right]^{T}$, and the resulting trajectories of agent 1 are depicted by blue lines. Therefore, the system is asymptotically stable under the proposed control law.

With the above results in hand, to attenuate the bounded time-varying disturbances, assume that $\left|d_{i}(t)\right| \leq \theta_{i} \varphi_{i}(t)$, where $\theta_{i}$ is unknown constant, $\varphi_{i}(t)$ is bounded function of time $t$, the robust controller is derived by the following procedure:

$$
V_{t d}=\sum_{j \in N_{i}} \frac{1}{2}\left(a_{i j} e_{q i}^{2}+\frac{1}{2} \beta_{i j} e_{p i}^{2}+\frac{1}{2} \widetilde{\theta}_{i} \gamma_{i}^{-1} \widetilde{\theta}_{i}\right)
$$

where $\widehat{\theta}_{i}$ is an estimate of $\theta_{i}, \widetilde{\theta}=\theta-\widehat{\theta}$ is the parameter error; $\alpha_{i j}, \beta_{i j}$, and $\gamma_{i}$ are positive constants. Equation (48) is continuously differentiable. The time derivative of $V_{t a}$ is

$$
\begin{aligned}
\dot{V}_{t d} & =\sum_{j \in N_{i}}\left(a_{i j} e_{q i} \dot{e}_{q i}+\beta_{i j} e_{p i} \dot{e}_{p i}+\tilde{\theta}_{i} \gamma_{i}^{-1} \dot{\tilde{\theta}}\right) \\
& =\sum_{j \in N_{i}}\left(a_{i j} e_{q i} e_{p i}+\beta_{i j} e_{p i} u_{i}+\beta_{i j} e_{p i} d_{i}+\tilde{\theta}_{i} \gamma_{i}^{-1} \dot{\tilde{\theta}}\right) \\
& =\sum_{j \in N_{i}}\left(e_{q i} e_{p i}+\beta_{i j} e_{p i} u_{i}+\beta_{i j} e_{p i} \theta_{i} \varphi(t)+\tilde{\theta}_{i} \gamma_{i}^{-1} \dot{\tilde{\theta}}\right)
\end{aligned}
$$

Design the adaptive control $u_{i}$ as

$$
u_{i}=\sum_{j \in N_{i}}\left(-\frac{a_{i j}}{\beta_{i j}} e_{q i}-e_{p i}-\widehat{\theta}_{i} \varphi(t)\right)
$$

Substituting (50) into (49), we have

$$
\dot{V}_{t d}=\sum_{j \in N_{i}}\left(-\beta_{i j} e_{p i}^{2}+\beta_{i j} e_{p i}\left(\theta_{i}-\widehat{\theta}_{i}\right) \varphi(t)+\widetilde{\theta}_{i} \gamma_{i}^{-1} \dot{\tilde{\theta}}\right)
$$



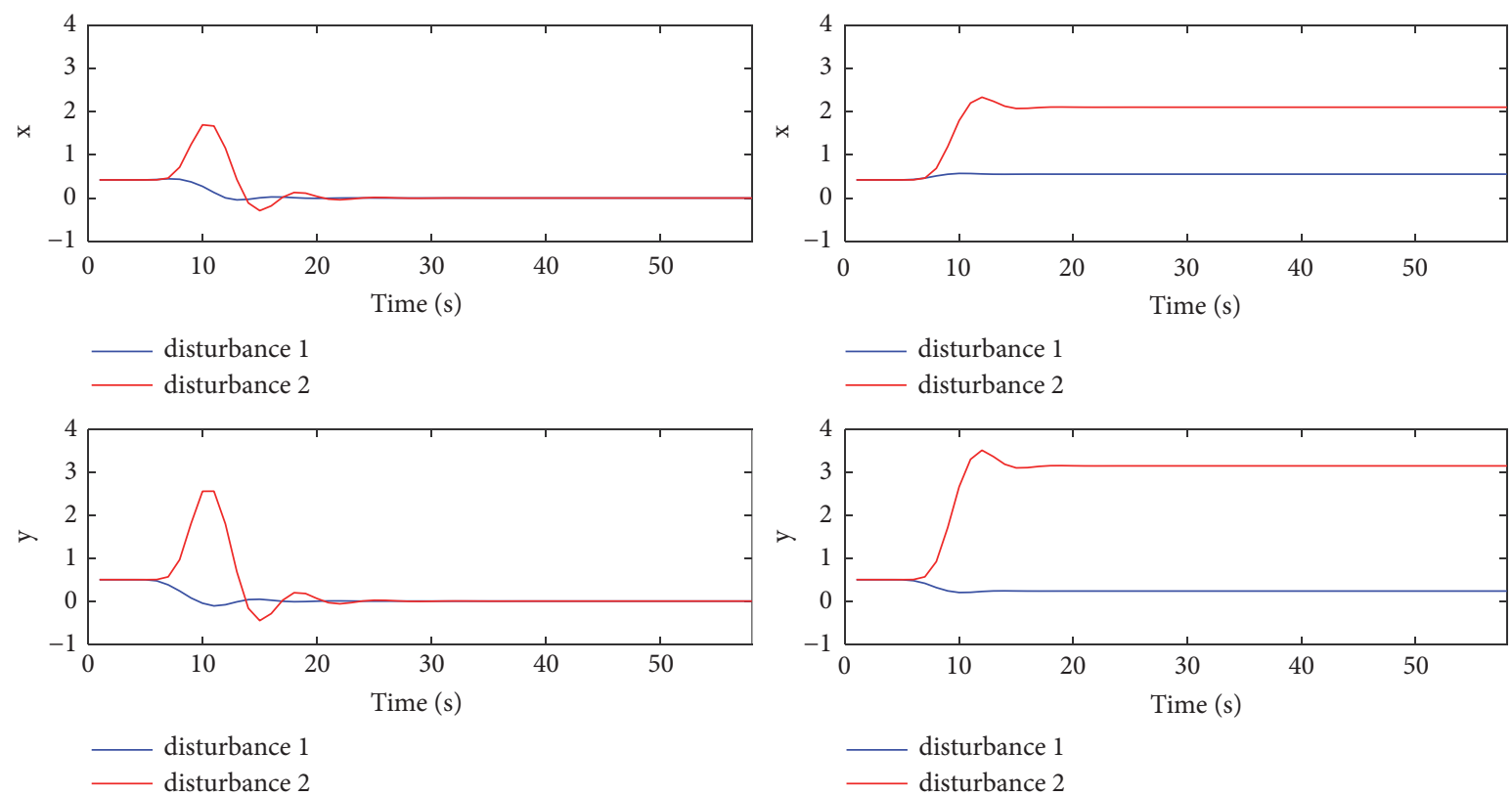

Figure 4: State trajectory of agent 1 in the presence of constant disturbances. Left: using the integral control, Right: without the help of the integral control for the disturbances.

To guarantee the negative semidefinite of $\dot{V}_{t d}$, the adaptation law is chosen as

$$
\dot{\tilde{\theta}}=-\gamma_{i} \beta_{i j} e_{p i} \varphi(t)-\gamma_{i}\left(\theta_{i}-\widehat{\theta}_{i}\right)
$$

Substituting (52) into (51) yields

$$
\dot{V}_{t d}=\sum_{j \in N_{i}}\left(-\beta_{i j} e_{p i}^{2}-\left(\theta_{i}-\widehat{\theta}_{i}\right)^{T}\left(\theta_{i}-\widehat{\theta}_{i}\right)\right)
$$

which is continuously differentiable function and negative semi-definite, implying that $\mathrm{V}_{t d}(t)-\mathrm{V}_{t d}(0) \leq 0$, with the equality is satisfied if and only if $e_{p i}=0$ and $\widehat{\theta}_{i}=\theta_{i}$; it follows that $e_{p i}=0$. Therefore, the system is asymptotically stable, the desired formation is achieved, and the time-varying disturbances are attenuated under the adaptive control law (50).

\section{Simulation Results}

In this section, numerical simulation results are given to support the obtained theoretical results. Consider a fiveagent system under the leader-follower structure as shown in Figure 1. tem as

Consider the second-order dynamics for multiagent sys-

$$
\begin{aligned}
& \dot{q}_{i}=p_{i}, \\
& \dot{p}_{i}=\left[\begin{array}{c}
-2 q_{i 1}-3 p_{i 1} \\
-2 q_{i 2}-3 p_{i 2}
\end{array}\right]+\left[\begin{array}{l}
0.52 p_{i 1} \\
4+p_{i 2}
\end{array}\right] u_{i}+d_{i}(t)
\end{aligned}
$$

To characterize the desired formation, the desired relative position vector is provided, i.e., $q_{1}^{d}=\left[\begin{array}{ll}4 & 0\end{array}\right]^{T} ; q_{2}^{d}=\left[\begin{array}{ll}2 & 2\end{array}\right]^{T}$; $q_{3}^{d}=\left[\begin{array}{ll}2 & -2\end{array}\right]^{T} ; q_{4}^{d}=\left[\begin{array}{ll}-2 & 2\end{array}\right]^{T} ; q_{5}^{d}=\left[\begin{array}{ll}-2 & -2\end{array}\right]^{T}$. The initial positions of the agents are randomly given as $q_{1}(0)=$ $\left[\begin{array}{ll}0.42 & 0.50\end{array}\right]^{T}, q_{2}(0)=\left[\begin{array}{ll}0.35 & -0.67\end{array}\right]^{T}, q_{3}(0)=\left[\begin{array}{ll}0.04 & -0.46\end{array}\right]^{T}$, $q_{4}(0)=\left[\begin{array}{ll}0.12 & -0.06\end{array}\right]^{T}$, and $q_{5}(0)=\left[\begin{array}{ll}0.18 & 0.19\end{array}\right]^{T}$, which is illustrated in Figure 5. The other control parameters are chosen as $\gamma_{4}=\left[\begin{array}{ll}1.46 & 2.73\end{array}\right]$ and $\beta_{4}=\left[\begin{array}{ll}1.53 & 2.04\end{array}\right]$, and the external bounded time-varying disturbance $d_{4}(t)=$ $[5.17 \cos (0.75 t) 8.68 \sin (0.38 t)]$ is imposed on the $4^{\text {th }}$ agent as time evolves, without loss of generality and for brevity. Note that obstacle avoidance problem is considered in the simulation examples.

To proceed, the desired trajectory for the leader is then provided as

$$
\begin{aligned}
& \dot{q}_{r}=p_{r}, \\
& \dot{p}_{r}=\left\{\begin{array}{l}
0.12 \cos \left(0.15 p_{r}(2)\right) \\
0.26 .
\end{array}\right.
\end{aligned}
$$

Without loss of generality and for simplicity, the Laplacian matrix is defined as

$$
L=\left[\begin{array}{ccccc}
1 & 0 & 0 & 0 & 0 \\
-1 & 1 & 0 & 0 & 0 \\
-1 & -1 & 2 & 0 & 0 \\
0 & -1 & -1 & 2 & 0 \\
0 & 0 & -1 & -1 & 2
\end{array}\right]
$$

and the weight matrix $\Delta_{L}$ is given as $\Delta_{L}=\operatorname{diag}\{1,1,0,0,0\}$. It is easily verified that all the eigenvalues of $\left(L+\Delta_{L}\right)$ are positive. 


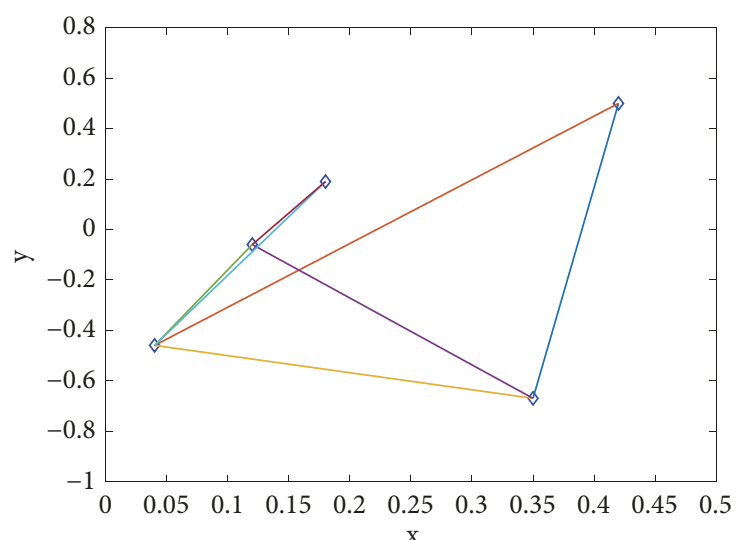

FIGURE 5: Initial positions of agents.

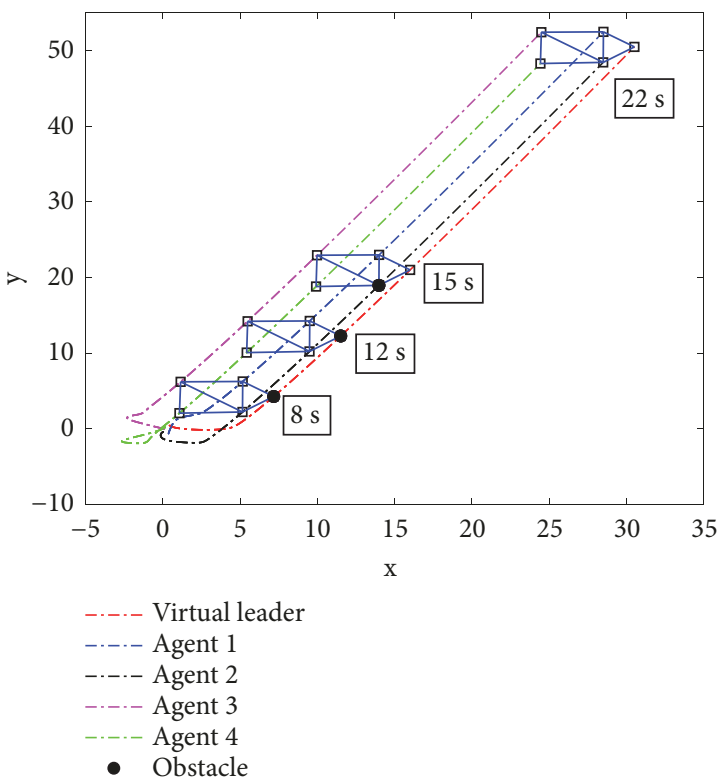

FIGURE 6: Formation trajectory without using the repulsive force.

The decentralized controller is given as

$$
\begin{aligned}
u_{i}= & \gamma_{i j}\left(\alpha_{i j}, y_{i j}\right)\left(q_{j}-q_{i}+q_{i}^{d}-q_{j}^{d}\right) \\
& +\gamma_{i k}\left(\alpha_{i k}, y_{i k}\right)\left(q_{k}-q_{i}+q_{i}^{d}\right)
\end{aligned}
$$

where $\gamma_{i}(\cdot, \cdot)$ remains unchanged and depends on the distance errors $y_{i j}$ and $y_{i k}$.

It is assumed that three obstacles points are considered and placed at $q_{o 1}=\left[\begin{array}{ll}7.18 & 4.25\end{array}\right]^{T}, q_{o 2}=\left[\begin{array}{ll}11.52 & 12.25\end{array}\right]^{T}$, and $q_{03}=\left[\begin{array}{ll}13.98 & 18.94\end{array}\right]^{T}$, respectively, which is shown in Figure 6. Figure 6 illustrates the formation trajectory for a group of agents, where the squares denote the final positions of the agent at each simulation instant. As seen, the desired formation cannot be achieved at $4 \mathrm{~s}$. As time $t$ evolves, the target formation is achieved and maintained
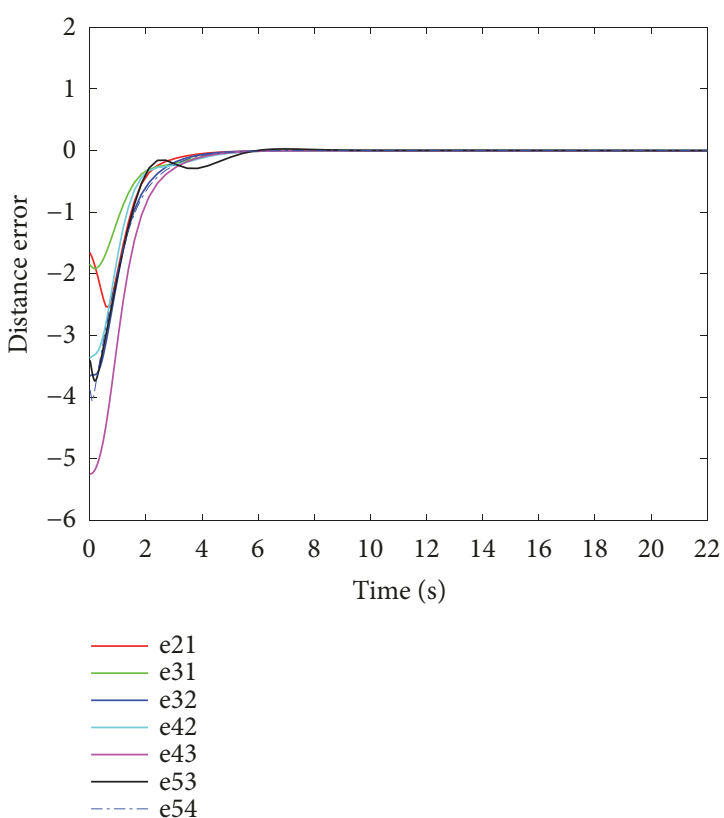

FIGURE 7: Errors between the actual distances and the desired distances without considering obstacle avoidance.

from at approximately $8 \mathrm{~s}$. The corresponding distance errors converge to zero as time $t$ evolves, which is shown in Figure 7.

As the agents sense each obstacle, the corresponding obstacle avoidance control term is then activated, which leads to the desired formation trajectory being changed. After avoiding the obstacle, the desired formation of five agents is rebuilt and achieved under the attractive force of the proposed formation control law at approximately $22 \mathrm{~s}$. Figure 8 depicts the formation for multiagent system approaches to three obstacles, respectively.

Figure 9 shows the distance errors between the actual distances and the corresponding desired distances among agents. Clearly, the distances errors tend to zero after avoiding each obstacle, implying that the actual distances converge to the corresponding desired distances as time $t$ evolves. The desired formation is therefore achieved. Moreover, we can see that the distance errors increase sharply when agents approach the obstacles, which implies that the repulsive potential field increases and steers the agents away from obstacles, as expected.

Without loss of generality, consider the multiagent system with constant bounded disturbance is given as

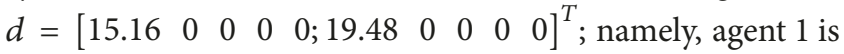
assumed to be disturbed in this setup.

The comparative results between without and with integral actions are given in Figures 10 and 11, respectively. The desired formation is not achieved and maintained in Figure 10, implying that the system is unstable, whereas the desired formation is achieved and maintained under the integral action, which is shown in Figure 11. For agent 1 , the static error $e 21$ is not eliminated 


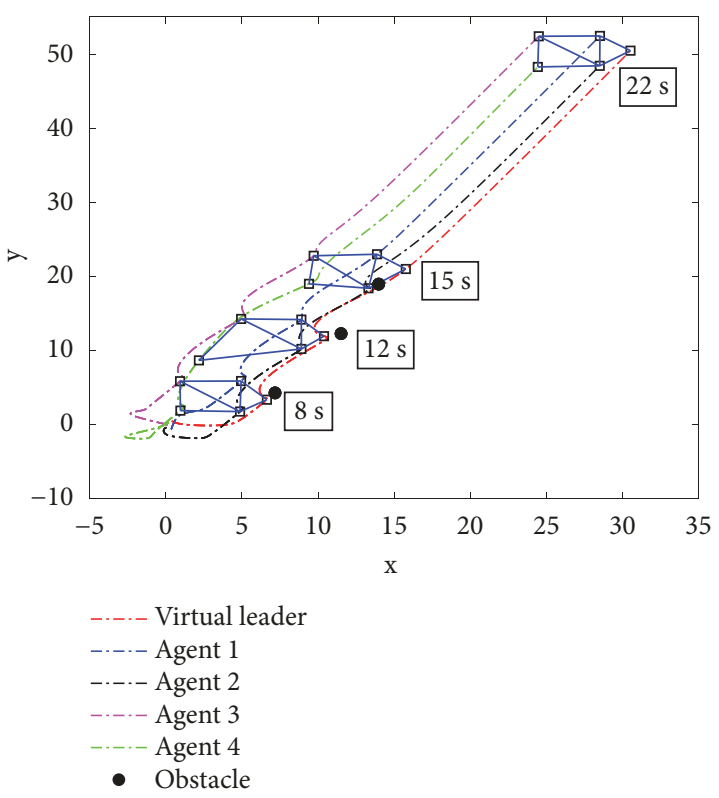

FIGURE 8: Formation control with obstacle avoidance by using the repulsive force.

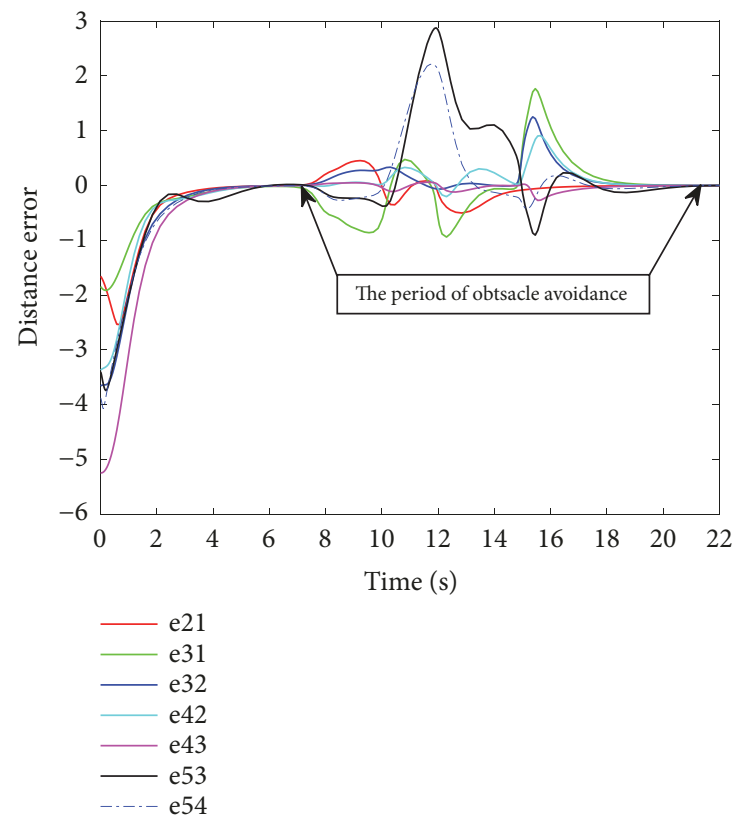

FIGURE 9: Distance errors of formation with obstacle avoidance. The period of obstacle avoidance is depicted.

without using the integral control term, which is illustrated in Figure 12. The static error $e 21$ can be eliminated with the aim of integral action, which is shown in Figure 13.

The comparative results between without and with adaptive control actions are given in Figures 14 and 15, respectively. The desired formation is not achieved and maintained in Figure 14, implying that the system is unstable, whereas

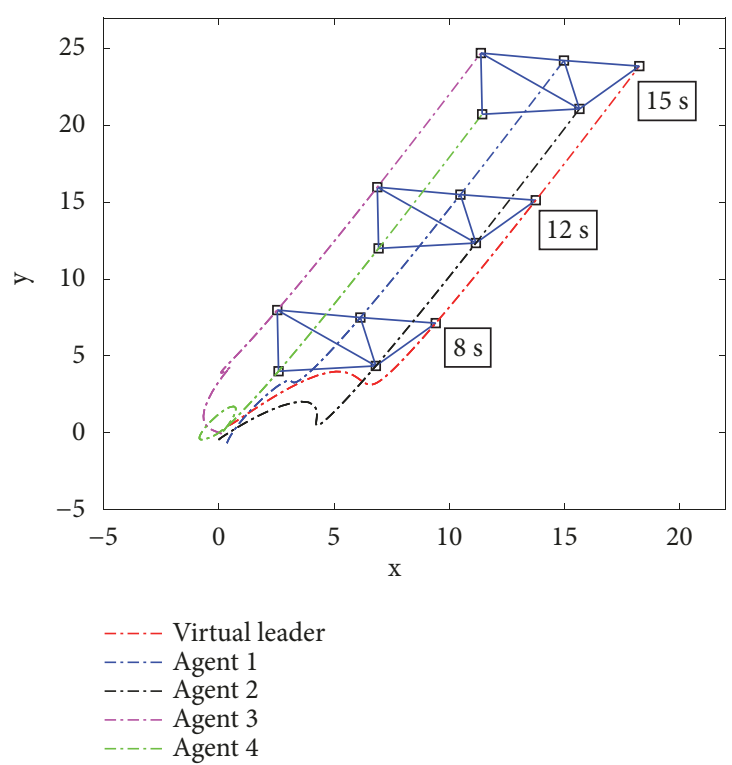

FIgURE 10: Formation trajectory in the presence of constant disturbance without employing the integral action.

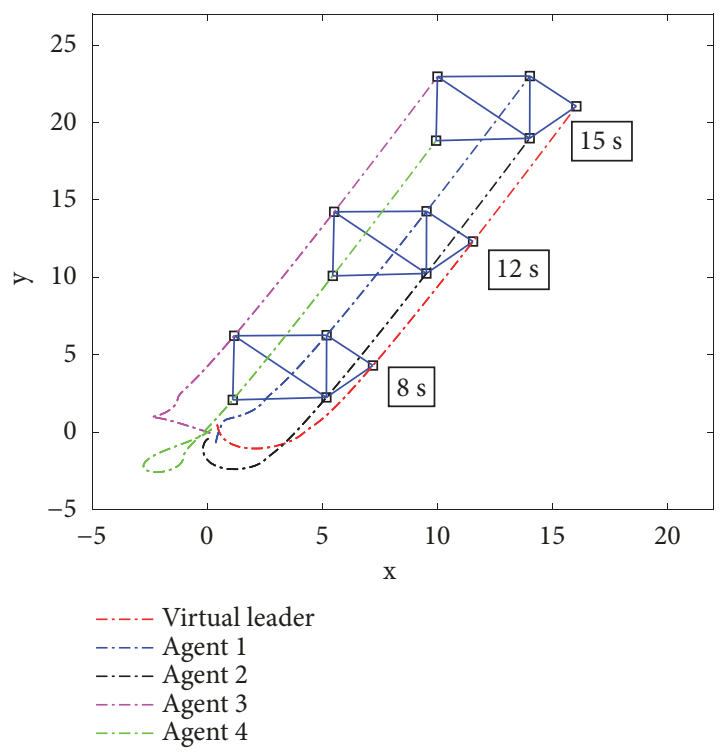

FIGURE 11: Formation trajectory in the presence of constant disturbance with the aim of integral action.

the desired formation is achieved and maintained under the adaptive control action, which is shown in Figure 15, which is further supported by the distance error shown in Figures 16 and 17, respectively. Figures 18 and 19 show the trajectories of estimates of parameters $\theta_{41}$ and $\theta_{42}$, and the curves of parameter estimation errors are depicted in Figure 20, which implies that the errors converge asymptotically to zero. Figure 21 shows the trajectory of the adaptation laws. 


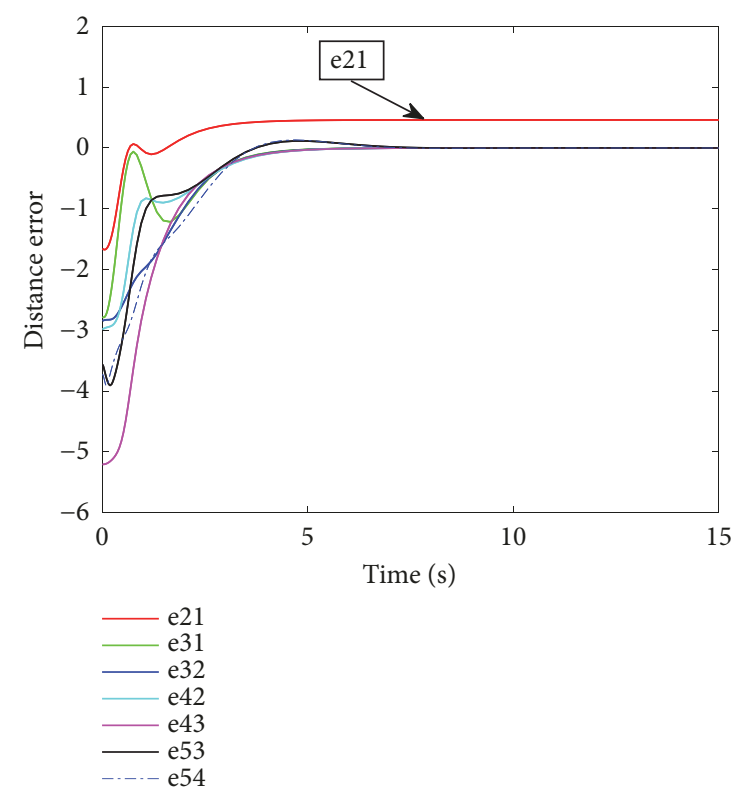

FIGURE 12: Distance errors in the presence of constant disturbance without integral action.

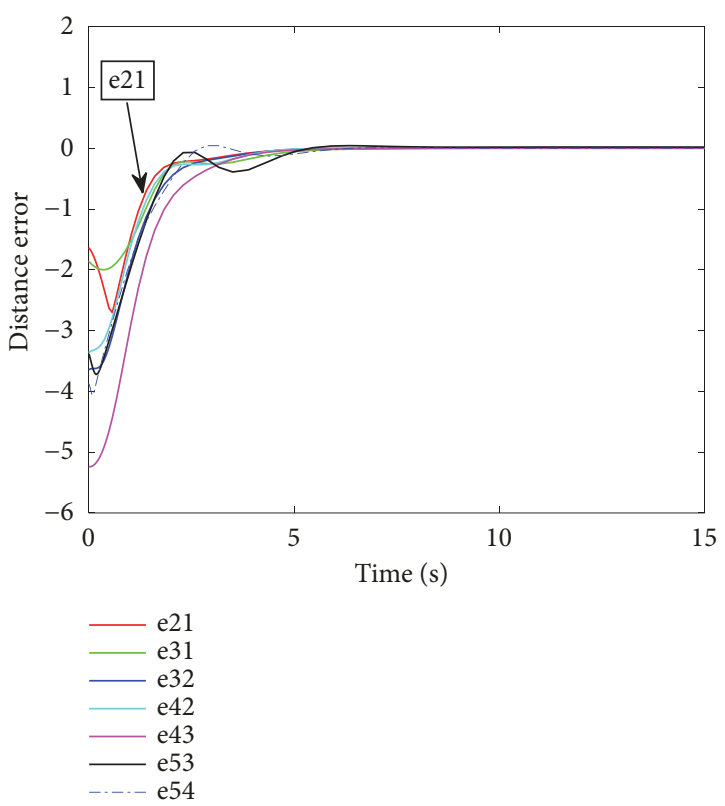

FIGURE 13: Distance errors in the presence of constant disturbance under the assistance of integral action.

\section{Conclusion}

A distributed nonlinear formation scheme was proposed for second-order dynamical systems, where the control input of each follower can be expressed as a product of a nonlinear term under the leader-follower structure. To deal with the obstacle problem, the artificial potential function approaches were applied to steer the agents away from the obstacles without getting stuck in local minimum positions. The stability analysis for a multiagent system is proved based on

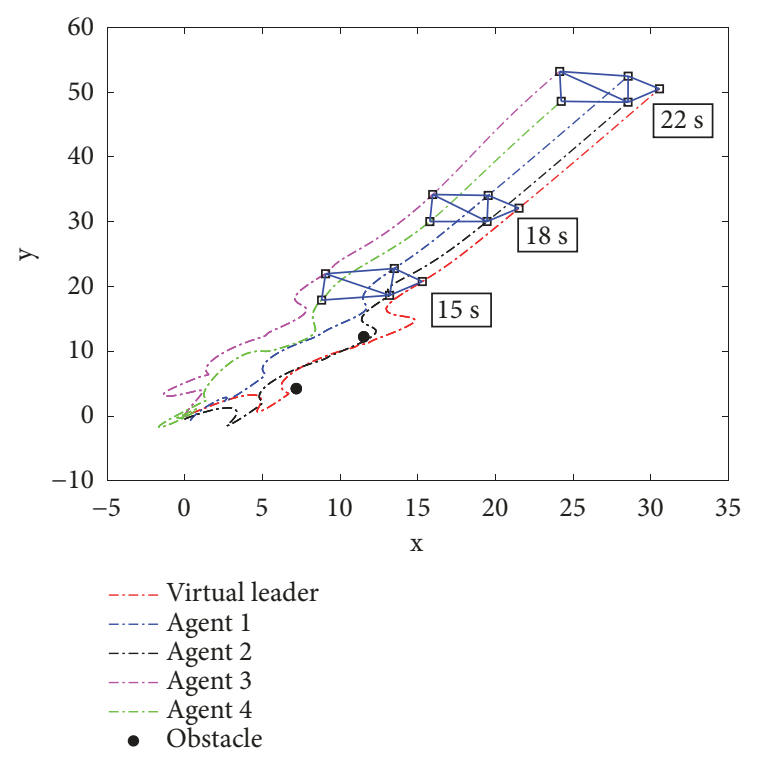

FIGURE 14: Formation trajectory in the presence of time-varying disturbance without employing the adaptive control term.

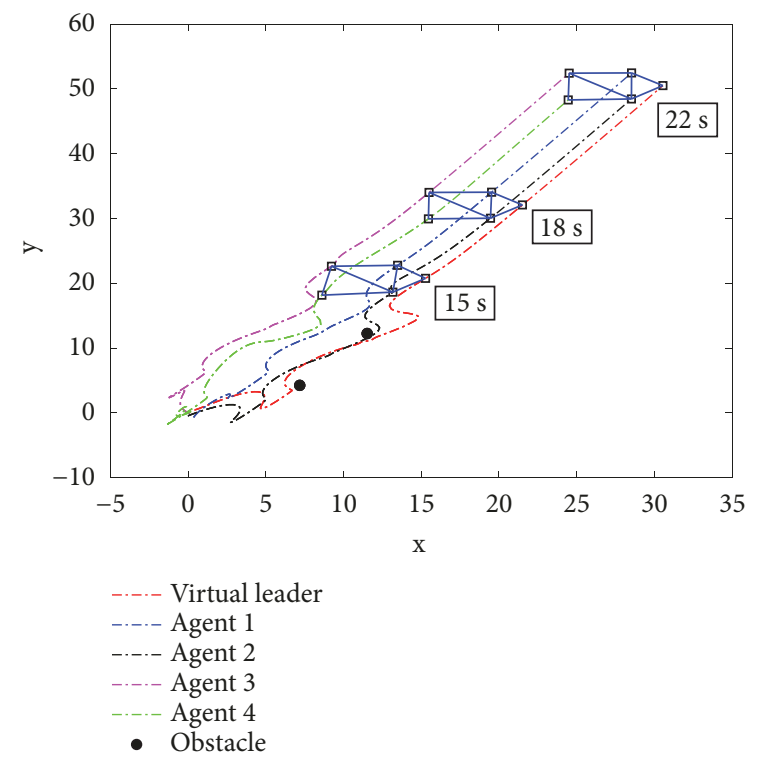

FIGURE 15: Formation trajectory in the presence of time-varying disturbance with the aim of the adaptive control term.

the theory of Lyapunov stability. Using the proposed control law, the desired formation is achieved and maintained while avoiding the obstacles. The formation control for multiagent systems in the presence of unknown bounded disturbances is investigated as well. Finally, the numerical simulation results are provided to demonstrate the effectiveness of the proposed distributed strategies.

\section{Data Availability}

The data used to support the finds of this article are included within the article. 

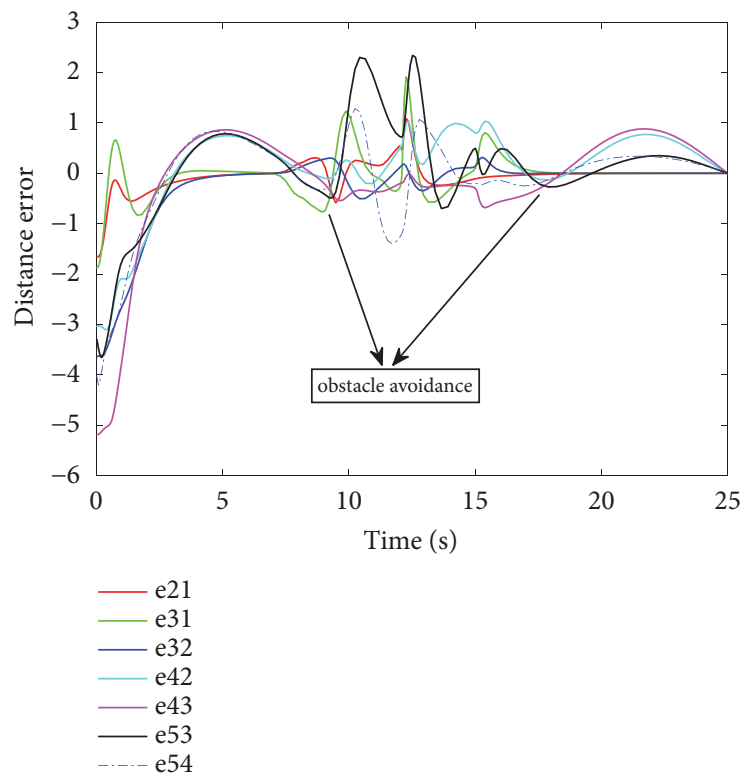

FIGURE 16: Distance errors in the presence of time-varying disturbance without the adaptive control term.
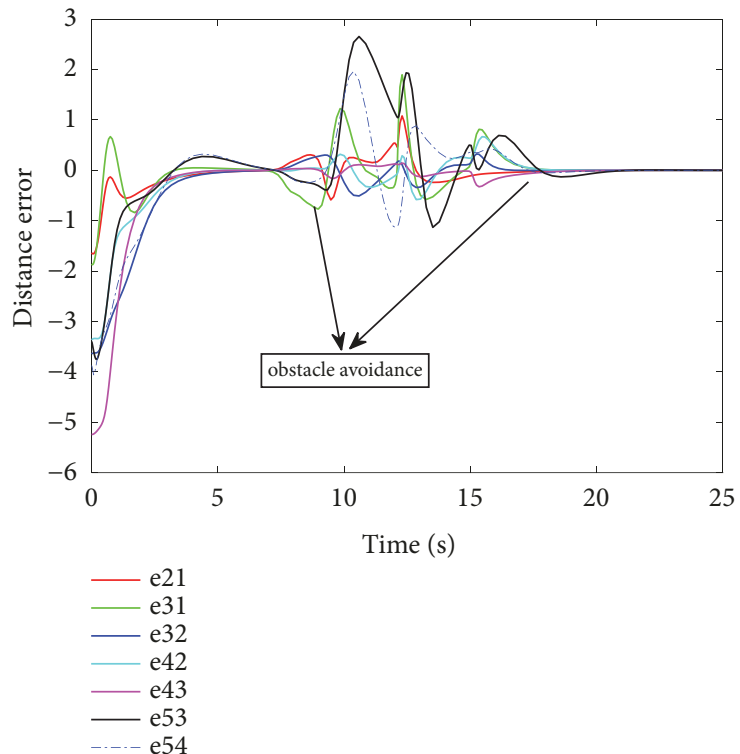

FIGURE 17: Distance errors in the presence of time-varying disturbance under the assistance of the adaptive control term.

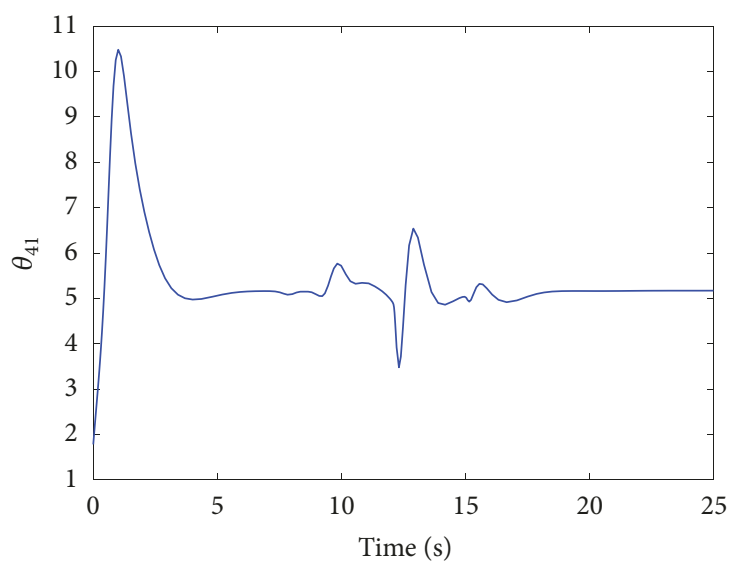

FIgURE 18: The estimate of parameter $\theta_{41}$. 


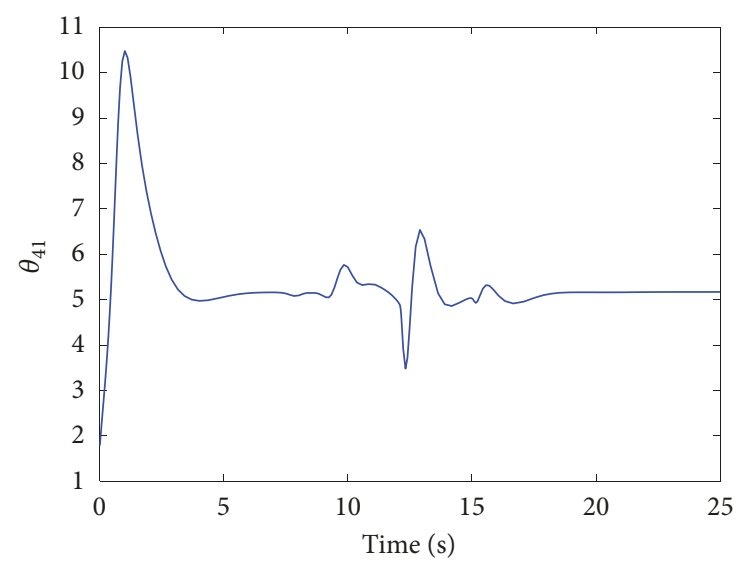

FIGURE 19: The estimate of parameter $\theta_{42}$.
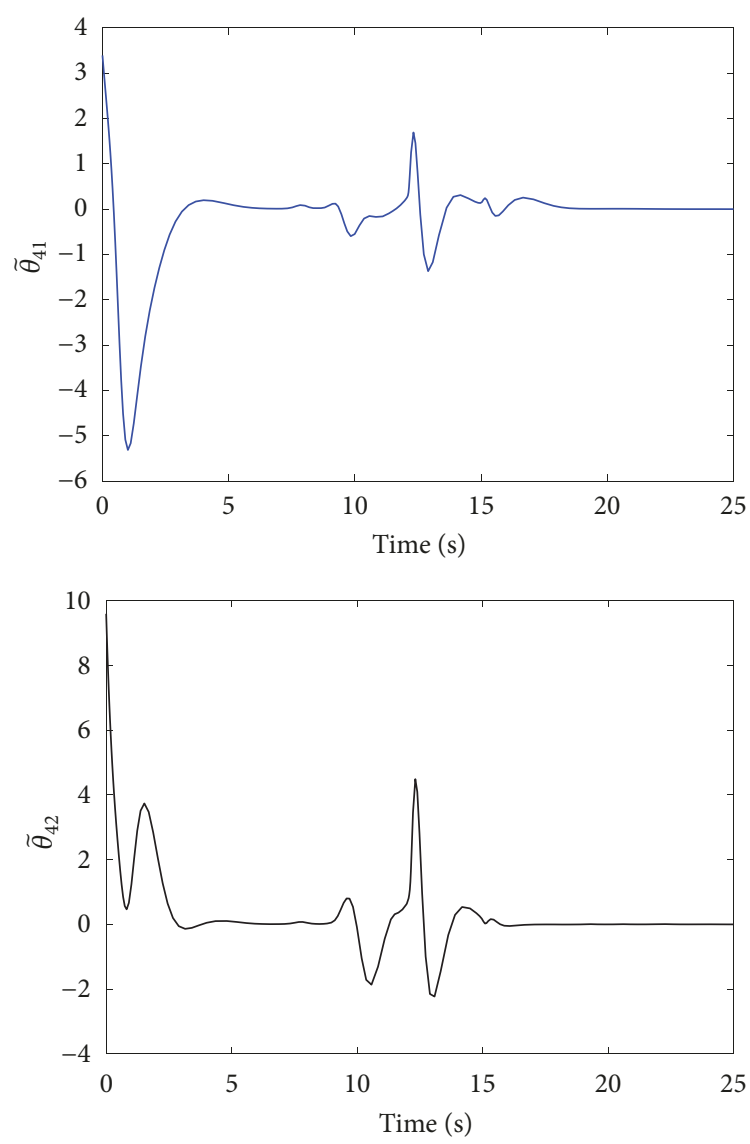

Figure 20: The parameter estimation errors.

\section{Conflicts of Interest}

The authors declare that they have no conflicts of interest regarding the publication of this paper.

\section{Acknowledgments}

This work was supported by the National Natural Science Foundation of China under Grant Nos. 61402540, 60903222,
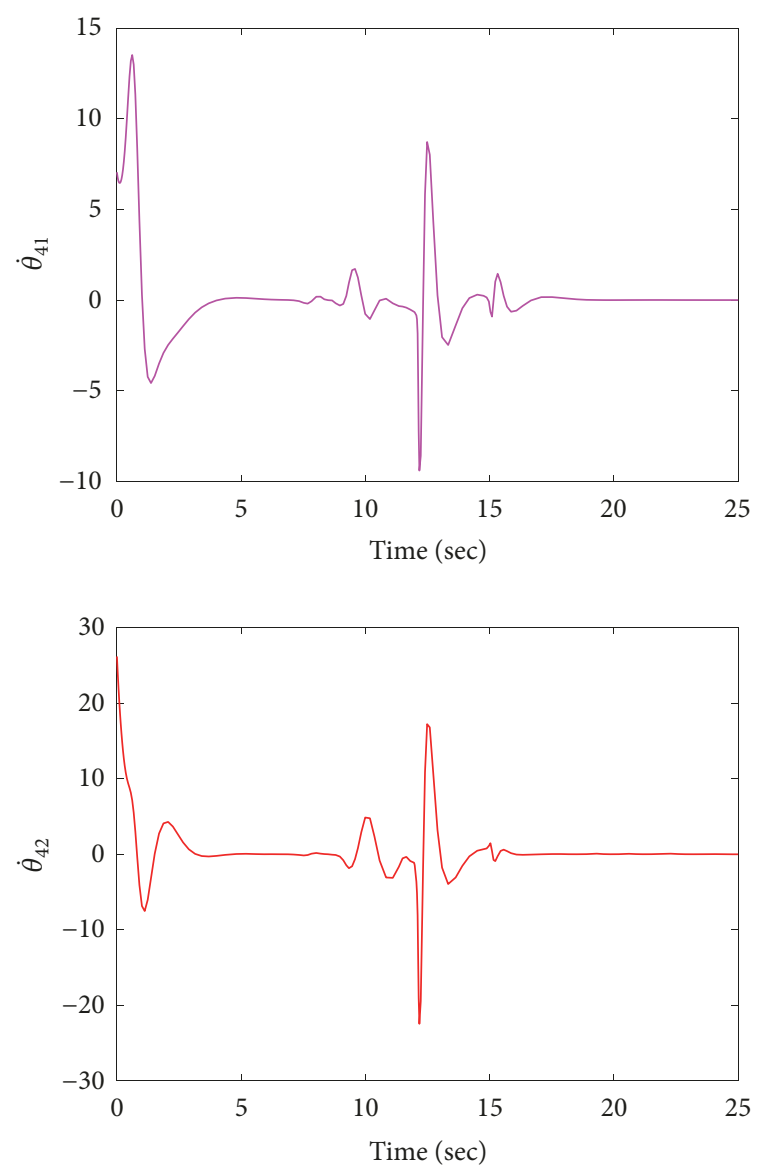

Figure 21: Adaptation laws.

61672538, and 61272024 and Hunan Provincial Science and Technology Foundation No. 2014GK3049.

\section{References}

[1] Z. Y. Wang and D. B. Gu, "Cooperative target tracking control of multiple robots," IEEE Transactions on Industrial Electronics, vol. 59, no. 8, pp. 3232-3240, 2012.

[2] I. Bayezit and B. Fidan, "Distributed cohesive motion control of flight vehicle formations," IEEE Transactions on Industrial Electronics, vol. 60, no. 12, pp. 5763-5772, 2013.

[3] M. Iacono and A. Sgorbissa, "Path following and obstacle avoidance for an autonomous UAV using a depth camera," Robotics and Autonomous Systems, vol. 106, pp. 38-46, 2018.

[4] H. Liu, X. Dong, F. L. Lewis, and Y. Wan, "Robust formation control for multiple quadrotors subject to nonlinear dynamics and disturbances," in Proceedings of the 2018 IEEE 14th International Conference on Control and Automation (ICCA), pp. 5862, Anchorage, AK, USA, June 2018.

[5] L. Sun, W. Huo, and Z. Jiao, "Adaptive backstepping control of spacecraft rendezvous and proximity operations with input saturation and full-state constraint," IEEE Transactions on Industrial Electronics, vol. 64, no. 1, pp. 480-492, 2017.

[6] Z. Han, K. Guo, L. Xie, and Z. Lin, "Integrated relative localization and leader-follower formation control," Institute of Electrical and Electronics Engineers Transactions on Automatic Control, vol. 64, no. 1, pp. 20-34, 2019. 
[7] C. Yuan, H. He, and C. Wang, "Cooperative deterministic learning-based formation control for a group of nonlinear uncertain mechanical systems," IEEE Transactions on Industrial Informatics, vol. 15, no. 1, pp. 319-333, 2019.

[8] H. Rezaee and F. Abdollahi, "A decentralized cooperative control scheme with obstacle avoidance for a team of mobile robots," IEEE Transactions on Industrial Electronics, vol. 61, no. 1, pp. 347-354, 2014.

[9] K.-K. Oh, M.-C. Park, and H.-S. Ahn, "A survey of multi-agent formation control," Automatica, vol. 53, pp. 424-440, 2015.

[10] S.-M. Kang and H.-S. Ahn, "Design and realization of distributed adaptive formation control law for multi-agent systems with moving leader," IEEE Transactions on Industrial Electronics, vol. 63, no. 2, pp. 1268-1279, 2016.

[11] M. Andreasson, D. V. Dimarogonas, H. Sandberg, and K. H. Johansson, "Distributed control of networked dynamical systems: static feedback, integral action and consensus," IEEE Transactions on Automatic Control, vol. 59, no. 7, pp. 1750-1764, 2014.

[12] G. Lee and D. Chwa, "Decentralized behavior-based formation control of multiple robots considering obstacle avoidance," Intelligent Service Robotics, vol. 11, no. 1, pp. 127-138, 2018.

[13] T. Nguyen, H. M. La, T. D. Le, and M. Jafari, "Formation control and obstacle avoidance of multiple rectangular agents with limited communication ranges," IEEE Transactions on Control of Network Systems, vol. 4, no. 4, pp. 680-691, 2017.

[14] N. Senroy, G. T. Heydt, and V. Vittal, "Decision tree assisted controlled islanding," IEEE Transactions on Power Systems, vol. 21, no. 4, pp. 1790-1797, 2006.

[15] Z. Sun and B. D. O. Anderson, "Rigid formation control with prescribed orientation," in Proceedings of the IEEE International Symposium on Intelligent Control, ISIC 2015, pp. 639-645, Australia, September 2015.

[16] W. Ren, "Multi-vehicle consensus with a time-varying reference state," Systems \& Control Letters, vol. 56, no. 7-8, pp. 474-483, 2007.

[17] S. Zhao and D. Zelazo, "Translational and scaling formation maneuver control via a bearing-based approach," IEEE Transactions on Control of Network Systems, vol. 4, no. 3, pp. 429-438, 2017.

[18] S. Zhao, D. V. Dimarogonas, Z. Sun, and D. Bauso, "A general approach to coordination control of mobile agents with motion constraints," IEEE Transactions on Automatic Control, vol. 63, no. 5, pp. 1509-1516, 2018.

[19] S. Zhao and D. Zelazo, "Bearing-based formation maneuvering," in Proceedings of the IEEE International Symposium on Intelligent Control, ISIC 2015, pp. 658-663, Australia, September 2015.

[20] B. Jiang, M. Deghat, and B. D. Anderson, "Simultaneous velocity and position estimation via distance-only measurements with application to multi-agent system control," Institute of Electrical and Electronics Engineers Transactions on Automatic Control, vol. 62, no. 2, pp. 869-875, 2017.

[21] K.-K. Oh and H.-S. Ahn, "Leader-follower type distancebased formation control of a group of autonomous agents," International Journal of Control, Automation, and Systems, vol. 15, no. 4, pp. 1738-1745, 2017.

[22] G. Hu, "Robust consensus tracking for an integrator-type multiagent system with disturbances and unmodelled dynamics," International Journal of Control, vol. 84, no. 1, pp. 1-8, 2011.
[23] M. Komareji, Y. Shang, and R. Bouffanais, "Consensus in topologically interacting swarms under communication constraints and time-delays," Nonlinear Dynamics, vol. 93, no. 3, pp. 1-14, 2018.

[24] W. Cao, J. Zhang, and W. Ren, "Leader-follower consensus of linear multi-agent systems with unknown external disturbances," Systems \& Control Letters, vol. 82, pp. 64-70, 2015.

[25] G. Wen, C. L. P. Chen, and Y.-J. Liu, "Formation control with obstacle avoidance for a class of stochastic multiagent systems," IEEE Transactions on Industrial Electronics, vol. 65, no. 7, pp. 5847-5855, 2018.

[26] Z. Sun, B. D. Anderson, M. Deghat, and H.-S. Ahn, "Rigid formation control of double-integrator systems," International Journal of Control, vol. 90, no. 7, pp. 1403-1419, 2017.

[27] M. H. Trinh, S. Zhao, Z. Sun, D. Zelazo, B. D. Anderson, and H.S. Ahn, "Bearing-based formation control of a group of agents with leader-first follower structure," Institute of Electrical and Electronics Engineers Transactions on Automatic Control, vol. 64, no. 2, pp. 598-613, 2019. 


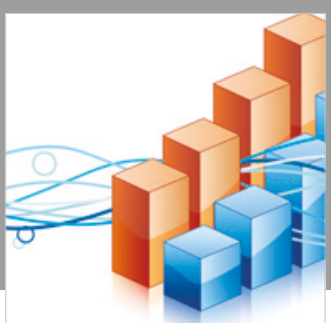

Advances in

Operations Research

\section{-n-m}
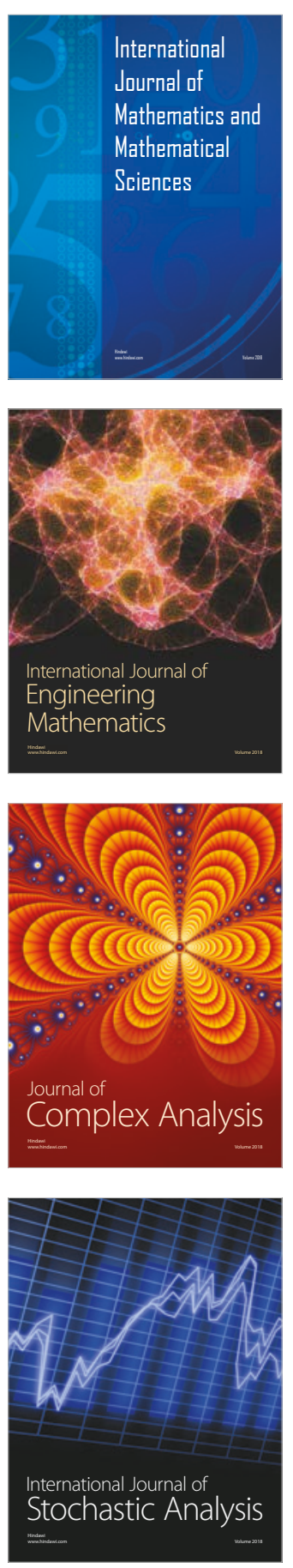
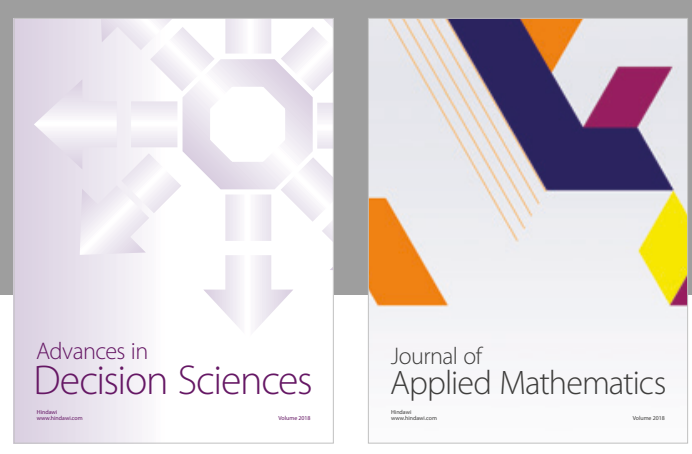

Journal of

Applied Mathematics
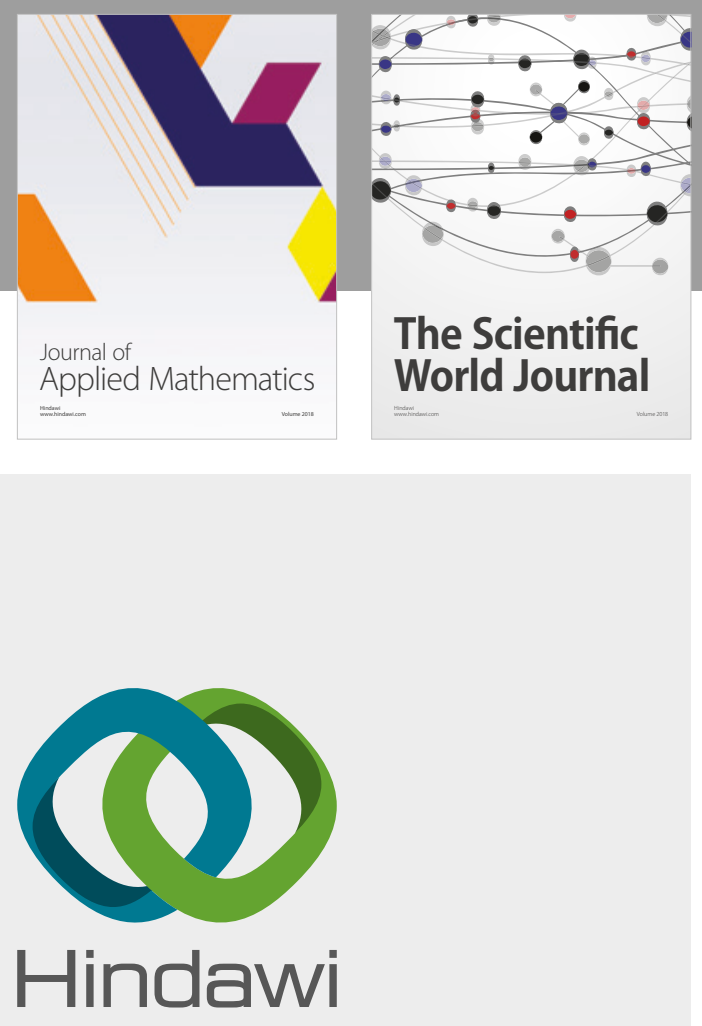

Submit your manuscripts at

www.hindawi.com

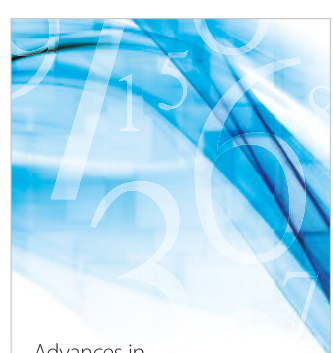

Advances in
Numerical Analysis
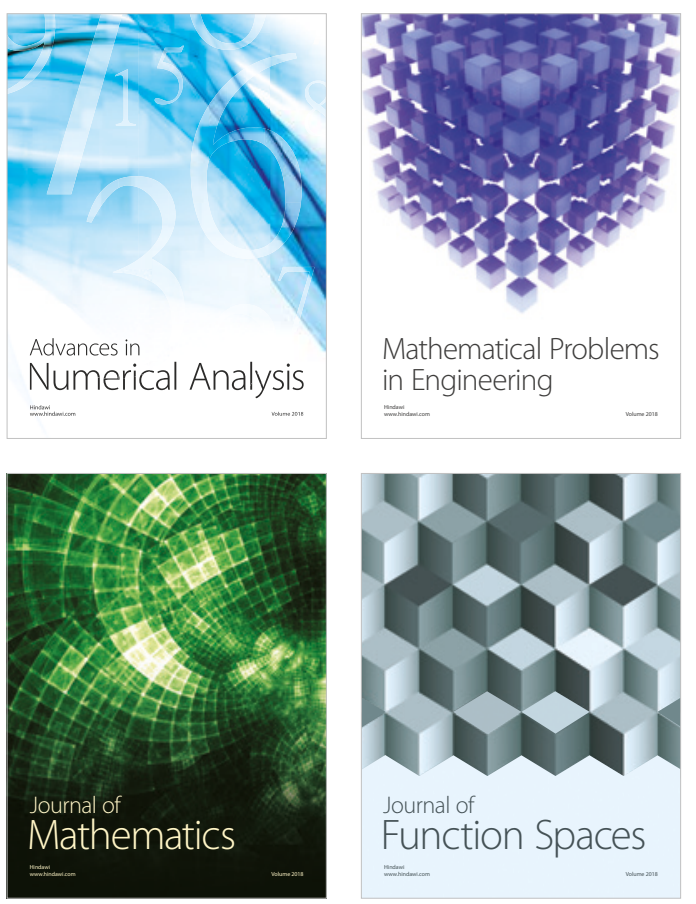

Mathematical Problems in Engineering

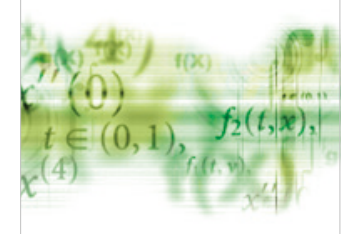

International Journal of

Differential Equations

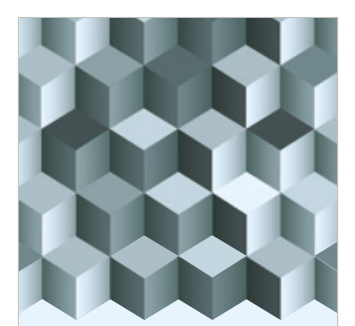

Journal of

Function Spaces
The Scientific

World Journal

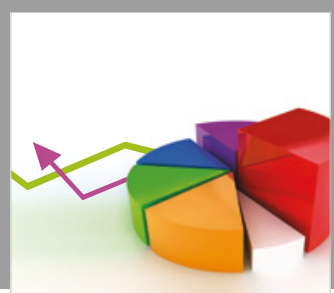

Journal of

Probability and Statistics
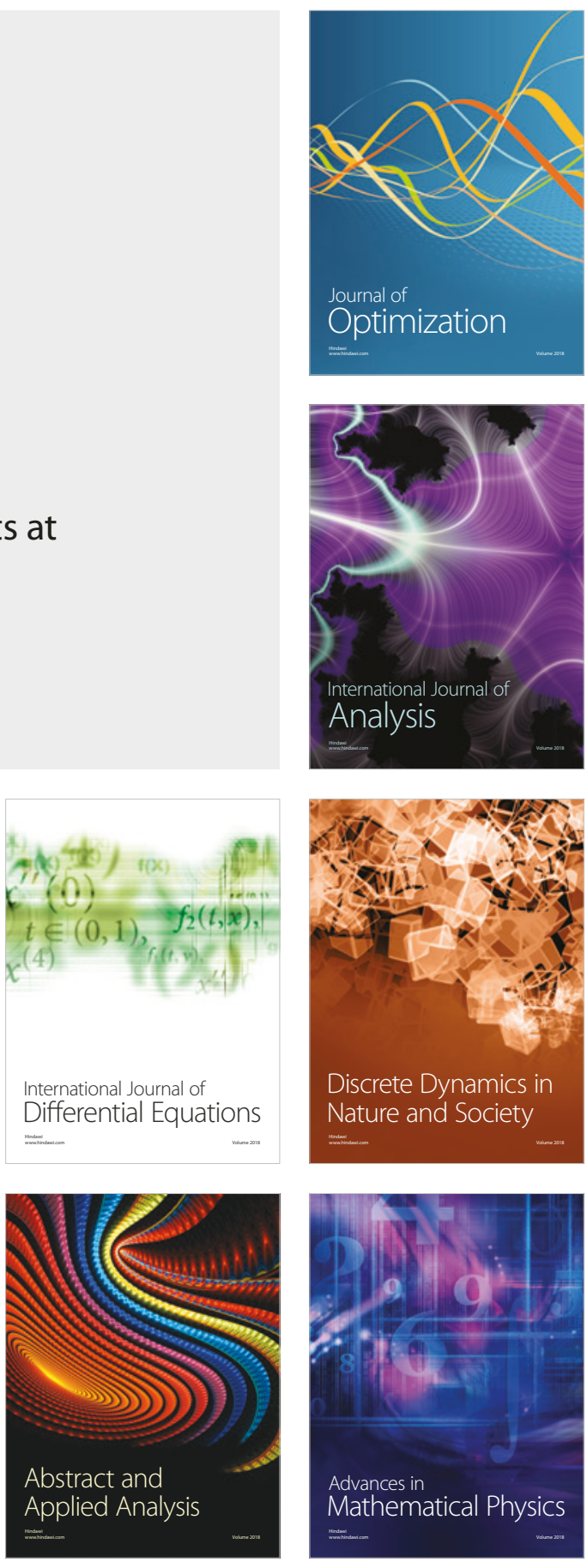